Archive for

Organic Chemistry

Arkivoc 2018, part iii, 212-228

\title{
Synthesis and anti-microbial / anti-malarial activity of a new class of chromone-dihydroquinazolinone hybrid heterocycles
}

\author{
Pavan Kumar Bathini, ${ }^{a}$ Hemasri Yerrabelly, ${ }^{b}$ and Jayaprakash Rao Yerrabelly*c,d \\ ${ }^{a}$ Department of Process Research \& Development, Dr. Reddy's Laboratories Limited, CTO-II, \\ Hyderabad 502 325, Telangana, India \\ ${ }^{b}$ Department of Chemistry, Nizam College, Osmania University-500 001, India \\ ${ }^{c}$ Department of Chemistry, Osmania University, Hyderabad, Telangana-500 007, India \\ ${ }^{d}$ Department of Chemistry, Telangana University, Nizamabad-503 322, India \\ E-mail:yipr19@yahoo.com
}

\section{Abstract}

A new series of chromone-2,3-dihydroquinazolin-4-one hybrid heterocycles are synthesized from chromone-2carbaldehydes by coupling with 2-aminoarylamides and hydrazides without oxidizing agents. The newly synthesized products exhibited moderate to good antimicrobial activity.
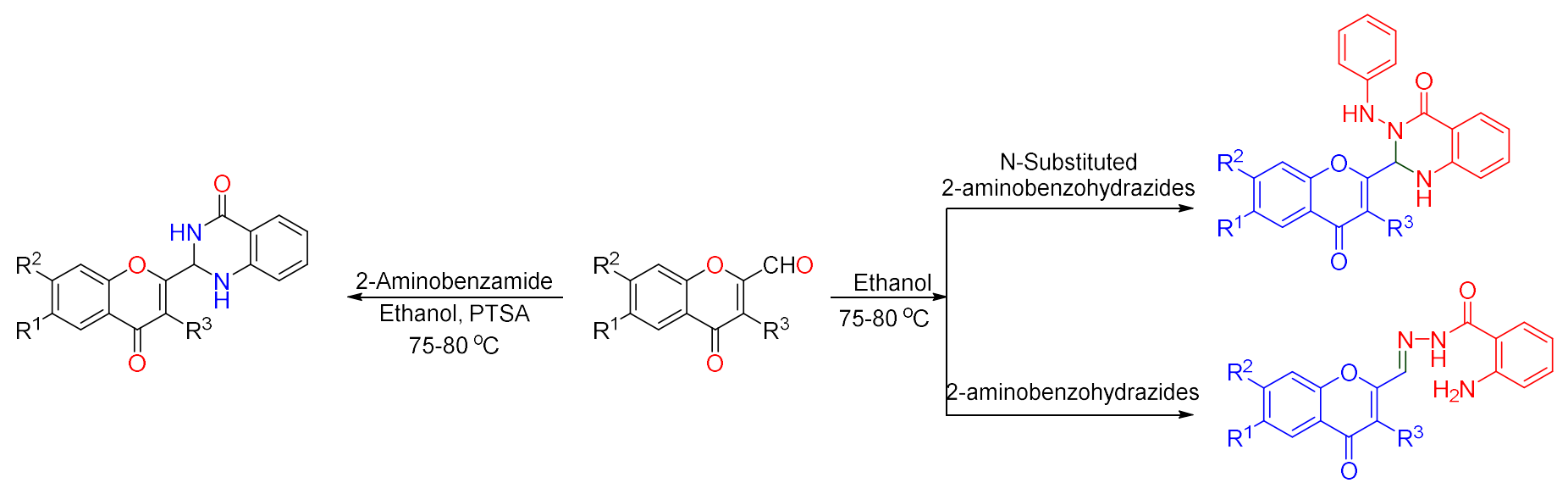

Keywords: Chromone-2-carbaldehydes, 2-aminobenzamide, 2-aminobenzohydrazide, 2,3-dihydroquinazolin-4ones, antimicrobial activity, antimalarial activity 


\section{Introduction}

Chromones and their derivatives are well-known naturally occurring oxygen heterocyclic compounds which exhibit important biological functions in nature. The rigid bicyclic chromone is a privileged moiety in drug discovery with activities such as anti-inflammatory, antitumor and anticancer activity. ${ }^{1-3}$ Simple, nonhydroxylated chromones were discovered to be selective inhibitors of p56lck tyrosine kinase. ${ }^{4}$ Thus, a simple, efficient synthesis of chromone molecular hybrids remains an important research topic.

Nitrogen-containing heterocyclic compounds are also reported to be significantly important because of their diverse biological and pharmacological activities such as antibacterial, antifungal, ${ }^{5-8}$ anticancer, ${ }^{9-11}$ antiparkinson, ${ }^{12}$ antidepressant, ${ }^{13}$ analgesic, ${ }^{14}$ diuretic ${ }^{15,16}$ and antihistamine activity. ${ }^{17}$ These compounds also act as vasodilating agents, ${ }^{18}$ antihypertensive, ${ }^{19,20}$ and CNS stimulant. ${ }^{21}$ Other major pharmacological activities include, antianxiety, ${ }^{22}$ tranquilizing, ${ }^{23}$ antifibrillatory, ${ }^{24}$ and anticonvulsant ${ }^{25}$ effects. Some of the important chromone and 2,3-dihydroquinazolin-4-one scaffold drug candidates are presented in Figure $1 .^{26-31}$

The heterocyclic molecular hybrids have advantages, such as, the potential to reduce the development of drug resistance and undesired side effects. ${ }^{32-39}$ Chromone scaffold based hybrid heterocyclic products exhibited a wide range of biological activity. ${ }^{40-45}$<smiles>COc1c2occc2c(OC)c2c(=O)cc(C)oc12</smiles>

Antiuretic 1<smiles>COc1cccc(C2NC(=O)c3cc(Cl)ccc3N2)c1</smiles>

Anticancer activity 4<smiles>O=c1cc(-c2ccc(O)cc2)oc2cc(O)cc(O)c12</smiles>

Anticancer

2<smiles>COc1cccc(C2NC(=O)c3ccccc3N2)c1</smiles>

Anti-inflammatory

5<smiles>O=C(O)c1cc(=O)c2c(OCC(O)COc3cccc4oc(C(=O)O)cc(=O)c34)cccc2o1</smiles>

Antiasthamatic 3<smiles>NS(=O)(=O)c1cc2c(cc1Cl)NC(c1ccccc1)NC2=O</smiles>

Diuretic activity 6

Figure 1. Chromone and 2,3-dihydroquinazolin-4-one drugs

In view of the interesting biological activities exhibited by chromones and 2,3-dihydroquinazolin-4-ones, structural modification of chromones has been attempted. The present work describes the design, synthesis and evaluation of antimicrobial and antimalarial activity of novel chromone-2,3-dihydroquinazolinone hybrid molecules. These novel scaffolds have been prepared by coupling 4-oxo-4H-chromene-2-carbaldehydes with 2-aminobenzamide and 2-aminobenzohydrazide derivatives. During the synthesis, formation of new intermediates has also been realized.

Literature survey has shown that while extensive studies have been carried out on chromone-3carbaldehyde ${ }^{46-47}$, there is not much work on the synthesis and chemistry of chromone-2-carbaldehydes, probably due to the complexity in synthetic procedures. 


\section{Results and Discussion}

4-Oxo-4H-chromen-2-carbaldehydes 9a-f were synthesized using reported procedures ${ }^{48-50}$ (1) by $\mathrm{SeO}_{2}$ oxidation of 2-methyl-4H-chromen-4-ones $7 \mathrm{a}-\mathrm{c},(2)$ by $\mathrm{MnO}_{2}$ oxidation of 2-(hydroxymethyl)-4H-chromen-4ones 8a-c (Scheme 1). Approach 2 was specific for 6-substituted chromones as approach 1 resulted in very low yields of compounds $9 e-f$.<smiles>[R]c1cc2oc(C)c([R])c(=O)c2cc1[R]</smiles>

7a-c
$\mathrm{SeO}_{2}$,

\section{1,4-dioxane}

a: $R^{1}=H \quad R^{2}=H \quad R^{3}=H$

b: $\mathrm{R}^{1}=\mathrm{H} \quad \mathrm{R}^{2}=\mathrm{H} \quad \mathrm{R}^{3}=\mathrm{Br}$

c: $\mathrm{R}^{1}=\mathrm{CH}_{3} \mathrm{R}^{2}=\mathrm{H} \quad \mathrm{R}^{3}=\mathrm{Br}$

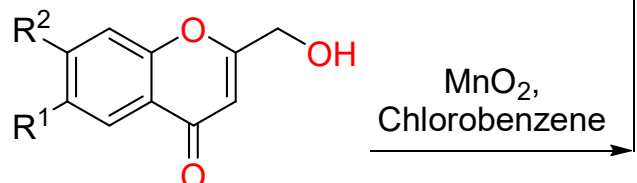

8a-c

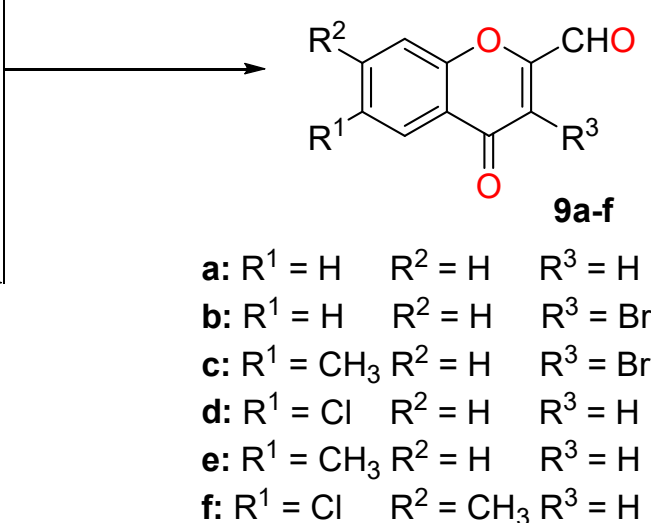
a: $\mathrm{R}^{1}=\mathrm{Cl} \quad \mathrm{R}^{2}=\mathrm{H}$
f: $\mathrm{R}^{1}=\mathrm{Cl} \quad \mathrm{R}^{2}=\mathrm{CH}_{3} \mathrm{R}^{3}=\mathrm{H}$

Scheme 1. Synthesis of 4-oxo-4H-chromene-2-carbaldehydes (9a-f)

4-Oxo-4H-chromene-2-carbaldehydes 9a-d were reacted with 2-aminobenzamide 10 in ethanol at 75-80 ${ }^{\circ} \mathrm{C}$ using PTSA as a catalyst for $3 \mathrm{hrs}$. The reaction was monitored for completion by TLC. The compounds 2-(4oxo-4H-chromen-2-yl)-2,3-dihydroquinazolin-4(1H)-ones 11a-d were isolated by column chromatography (Scheme 2). Under the present reaction conditions, formation of two compounds (the desired cyclized compound and an imine intermediate) was possible, but only the desired cyclized compounds 11a-d were obtained in excellent yields.<smiles>[R]c1cc2oc(C)c(CC)c(=O)c2cc1[R]</smiles>

9a-d<smiles>NC(=O)c1ccccc1N</smiles>

10

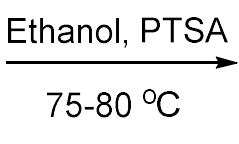
c: $\mathrm{R}^{1}=\mathrm{CH}_{3} \mathrm{R}^{2}=\mathrm{H}$
a: $R^{1}=H \quad R^{2}=H \quad R^{3}=H$
b: $R^{1}=H \quad R^{2}=H \quad R^{3}=B r$<smiles>[R]c1cc2oc(C3NC(=O)c4ccccc4N3)c([R])c(=O)c2cc1[R]</smiles>

11a-d

Scheme 2. Synthesis of 2-(4-oxo-4H-chromen-2-yl)-2,3-dihydroquinazolin-4(1H)-one (11a-d)

The IR (solid, $\mathrm{KBr}$ ) spectrum of 2-(4-oxo-4H-chromen-2-yl)-2,3-dihydroquinazolin-4(1H)-one 11a exhibited absorption bands at $3318 \mathrm{~cm}^{-1}(\mathrm{NH}), 1721 \mathrm{~cm}^{-1}$ (CO, chromone) and $1672 \mathrm{~cm}^{-1}$ (CO, amide). The ${ }^{1} \mathrm{H}$ NMR (400 $\left.\mathrm{MHz}, \mathrm{DMSO}-d_{6}\right)$ spectrum of the compound 11a showed ten signals corresponding to twelve protons. The aldehyde proton of 4-oxo-4H-chromen-2-carbaldehyde 9a was absent. Exchangeable protons were identified 
by $\mathrm{D}_{2} \mathrm{O}$ exchange analysis at $\delta_{\mathrm{H}} 8.67 \mathrm{ppm}\left(\mathrm{d}, J 2.8 \mathrm{~Hz}, 1 \mathrm{H}, \mathrm{NH}\right.$ amide) and $\delta_{\mathrm{H}} 7.5 \mathrm{ppm}$. Characteristic $\mathrm{CH}$ proton signals $(\mathrm{H}-2)$ appeared at $\delta_{\mathrm{H}} 5.76 \mathrm{ppm}(\mathrm{t}, J 2.8 \mathrm{~Hz}, 1 \mathrm{H})$. This observation suggested the formation of a cyclized compound 11a. The ${ }^{13} \mathrm{C}$ NMR (100 MHz, DMSO- $\left.d_{6}\right)$ spectrum of 11a showed seventeen signals. Of the seventeen signals, one was observed in the aliphatic region at $\delta_{c} 63.32 \mathrm{ppm}$, which can be correlated with the aliphatic proton at $\delta_{H} 5.76 \mathrm{ppm}$. The remaining 16 signals were observed between $\delta_{c} 108.07$ and $176.97 \mathrm{ppm}$. The positive-ion ESI-MS and HRMS data of 11a showed a protonated molecular ion at $m / z 293.09[\mathrm{M}+\mathrm{H}]^{+}$and $\mathrm{m} / \mathrm{z} 293.0936[\mathrm{M}+\mathrm{H}]^{+}$, respectively.

Following the above observations, the study was extended to 2-aminobenzohydrazides 14a-c which were synthesized using commercially available isatoic anhydride 12 and different hydrazine's 13a-c in DMF (Scheme 3).

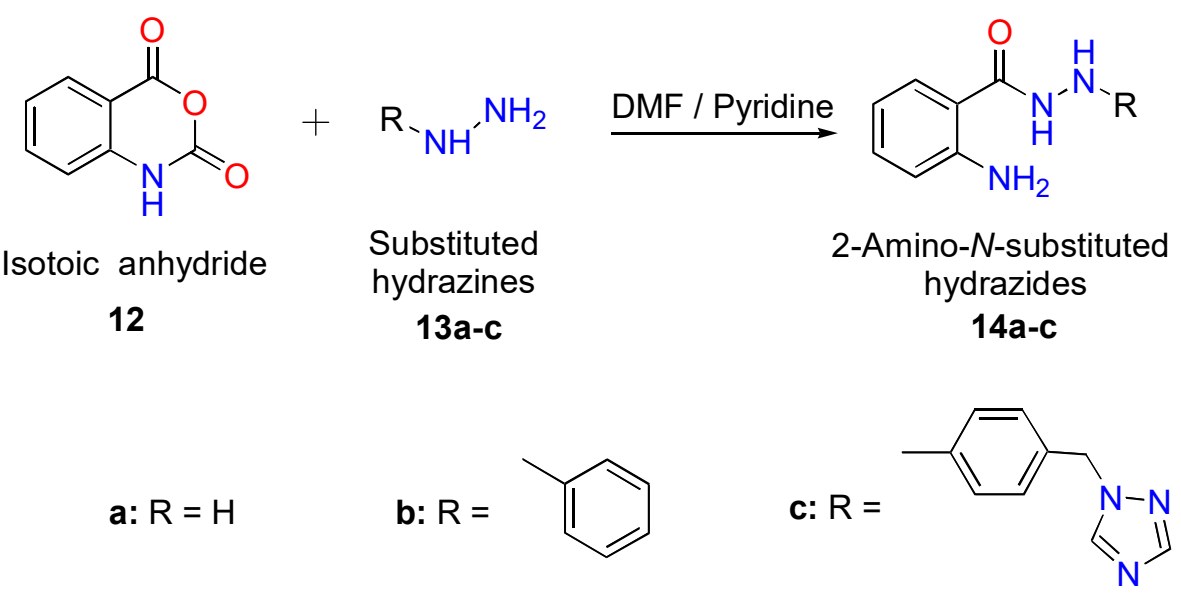

Scheme 3. Synthesis of 2-amino- $N$-substituted hydrazides (14a-c)

The reaction of 4-oxo-4H-chromene-2-carbaldehyde 9a-f with 2-aminobenzohydrazide 14a gave imine intermediates 15a-f and the desired cyclized products 16a-f were not observed even under forcing experimental conditions (Scheme 4). Literature survey supported the imine formation from aldehydes with 2aminobenzohydrazide. ${ }^{51}$

The IR (solid, $\mathrm{KBr}$ ) spectrum of 2-amino-N'-[(4-oxo-4H-chromen-2-yl)methylene]benzohydrazide 15a exhibited absorption bands at $3466 \mathrm{~cm}^{-1}\left(\mathrm{NH}\right.$, primary amine), $3351 \mathrm{~cm}^{-1}\left(\mathrm{NH}\right.$, secondary amine), $1738 \mathrm{~cm}^{-1}$ (CO, chromone) and $1663 \mathrm{~cm}^{-1}$ (CO, amide). The ${ }^{1} \mathrm{H}$ NMR (400 MHz, DMSO- $d_{6}$ ) spectrum of 15a showed twelve signals corresponding to thirteen protons. The aldehyde proton of $9 \mathrm{a}$ had disappeared. $\mathrm{D}_{2} \mathrm{O}$ exchange analysis showed exchangeable protons at $\delta_{\mathrm{H}} 12.2(\mathrm{~s}, 1 \mathrm{H}$, amide $\mathrm{NH})$ and $\delta_{\mathrm{H}} 6.52 \mathrm{ppm}\left(\mathrm{s}, 2 \mathrm{H}, \mathrm{NH}_{2}\right)$. Presence of two amine protons suggested the involvement of only the hydrazide $\mathrm{NH}_{2}$ in the reaction. The absence of a proton in the aliphatic region suggested that the compound formed was an imine, but not the expected cyclic product. The ${ }^{13} \mathrm{C}$ NMR (100 MHz, DMSO- $\mathrm{d}_{6}$ ) of 15a showed seventeen signals between $\delta_{c} 111.71 \mathrm{ppm}$ and $176.84 \mathrm{ppm}$. The absence of aliphatic carbons correlates with the absence of aliphatic protons in ${ }^{1} \mathrm{H}$ NMR. The positive ESI-MS and HRMS data of 15a showed the molecular ion at $m / z 308.1[\mathrm{M}+\mathrm{H}]^{+}$and $308.1046[\mathrm{M}+\mathrm{H}]^{+}$, respectively. From the HSQC data of 15a, the absence of correlations for the protons at $\delta_{\mathrm{H}} 12.2$ and $6.52 \mathrm{ppm}$ confirmed that these are not attached to any carbon. This data supported the $\mathrm{D}_{2} \mathrm{O}$ exchange information. The HSQC data showed that there are ten methine groups and seven quaternary carbons, two of them corresponding to carbonyl carbons. HOMO COSY of 15a showed two singlets at $\delta_{\mathrm{H}} 8.25$ and $6.72 \mathrm{ppm}$ which did not show any correlations. From the ${ }^{1} \mathrm{H}$ NMR study on 4-oxo-4H-chromene-2-carbaldehydes, the proton at $\delta_{H} 6.72 \mathrm{ppm}$ was assigned to the $\mathrm{H}-3$ olefin proton. The other proton at $\delta_{H} 8.25 \mathrm{ppm}$ which correlated with the 
carbon at $\delta_{c} 138.7 \mathrm{ppm}$ (HSQC) was predicted to be from an imine. This was supported by the double bond equivalence (DBE) from HRMS data.<smiles>[R]c1cc2oc(C=O)c([R])c(=O)c2cc1[R19]</smiles>

9a-f<smiles>[R]c1cc2oc(C3Nc4ccccc4C(=O)N3N)c([R])c(=O)c2cc1[R]</smiles>

16a-f not formed<smiles>Nc1ccccc1C(=O)N/N=C/c1cc(=O)c2ccccc2o1</smiles>

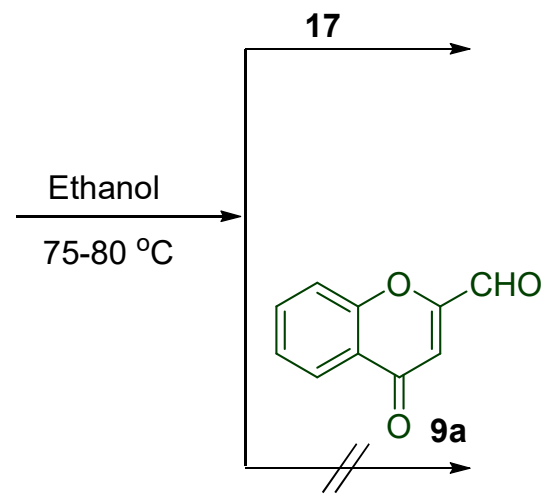<smiles>O=C1c2ccccc2NC(c2ccccc2)N1/N=C/c1cc(=O)c2ccccc2o1</smiles><smiles>O=C1c2ccccc2NC(c2cc(=O)c3ccccc3o2)N1/N=C/c1cc(=O)c2ccccc2o1</smiles>

$18 \mathrm{a}$
a: $\mathrm{R}^{1}=\mathrm{H} \quad \mathrm{R}^{2}=\mathrm{H} \quad \mathrm{R}^{3}=\mathrm{H}$
c: $\mathrm{R}^{1}=\mathrm{CH}_{3} \mathrm{R}^{2}=\mathrm{H} \quad \mathrm{R}^{3}=\mathrm{Br}$
e: $\mathrm{R}^{1}=\mathrm{CH}_{3} \quad \mathrm{R}^{2}=\mathrm{H} \quad \mathrm{R}^{3}=\mathrm{H}$
b: $\mathrm{R}^{1}=\mathrm{H} \quad \mathrm{R}^{2}=\mathrm{H} \quad \mathrm{R}^{3}=\mathrm{Br}$
d: $\mathrm{R}^{1}=\mathrm{Cl} \quad \mathrm{R}^{2}=\mathrm{H} \quad \mathrm{R}^{3}=\mathrm{H}$
f: $\mathrm{R}^{1}=\mathrm{Cl} \quad \mathrm{R}^{2}=\mathrm{CH}_{3} \mathrm{R}^{3}=\mathrm{H}$

Scheme 4. Synthesis of 2-amino-N'-[(4-oxo-4H-chromen-2-yl)methylene]benzohydrazide (15a-f)

The results can be explained based on the difference in nucleophilicity of the $\mathrm{NH}_{2}$ of hydrazide and aniline. The lone pair of electrons on the amino group corresponding to aniline 14a are conjugated with the aromatic nuclei and also the carbonyl group at the ortho position. Hence, these electrons are not freely available for the reaction when compared to the lone pair of electrons on the $\mathrm{NH}_{2}$ of hydrazide $14 a$.

The imine compound 15a was further reacted with benzaldehyde 17. Interestingly, the reaction yielded the novel cyclic product 3-\{[(4-oxo-4H-chromen-2-yl)methylene]amino\}-2-phenyl-2,3-dihydroquinazolin-4(1H)one 18 (Scheme 4). In the ${ }^{1} \mathrm{H}$ NMR spectrum of 18, the characteristic peak of cyclic compound $\mathrm{H}-2^{\prime}$ appeared at $\delta_{H} 6.67 \mathrm{ppm}$ and corresponding ${ }^{13} \mathrm{C}$ signal of $\mathrm{C}-2{ }^{\prime}$ appeared at $\delta_{\mathrm{C}} 76.32 \mathrm{ppm}$. When the same reaction was carried out using 4-oxo-4H-chromene-2-carbaldehyde 9a, the reaction did not occur (Scheme 4), probably due to steric hindrance.

Further reaction was carried out using the $N$-substituted 2-aminobenzohydrazides 14b,c. Interestingly, the compounds obtained were the desired cyclic products $19 a-f$ (Scheme 5 ). In the ${ }^{1} \mathrm{H}$ NMR (400 MHz, DMSO- $d_{6}$ ) 
spectra of the obtained compounds 19a-f, a characteristic signal of $\mathrm{H}-2$ was observed at $\delta_{\mathrm{H}} 5.93-6.41 \mathrm{ppm}$ and the corresponding carbon signal was observed at $\delta_{c} 71.4-72.2 \mathrm{ppm}$ in ${ }^{13} \mathrm{C} N M R$. In the case that the free $\mathrm{NH}_{2}$ of hydrazide 14a was substituted with a phenyl 14b (where the lone pair is conjugated with the phenyl ring), the $\mathrm{NH}$ of the amide participated in the reaction, resulting in cyclized compounds having two heterocyclic moieties.

In order to develop hybrid triazole heterocyclic skeletons 20a-d, 4-oxo-4H-chromene-2-carbaldehydes 9a-d were reacted with $N^{\prime}-\{4-[(1 H-1,2,4-$ triazol-1-yl)methyl] phenyl\}-2-aminobenzohydrazide 14c (Scheme 5).<smiles>[R]c1cc2oc(C=O)c([R])c(=O)c2cc1[R]</smiles>

9a-f<smiles>Nc1ccccc1C(=O)NNc1ccccc1</smiles>

$14 b$

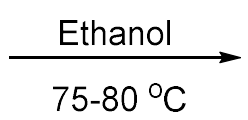

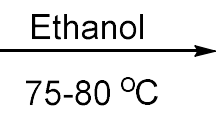

$14 \mathrm{c}$<smiles>Nc1ccccc1C(=O)NNc1ccc(Cn2cncn2)cc1</smiles><smiles>[R]c1cc2oc(C3Nc4ccccc4C(=O)N3Nc3ccccc3)c([R])c(=O)c2cc1[R]</smiles>

19a-f<smiles>[R]c1cc2oc([C@@H]3Nc4ccccc4C(=O)N3Nc3ccc(Cn4cncn4)cc3)c([R])c(=O)c2cc1[R]</smiles>

20a-d
c: $\mathrm{R}^{1}=\mathrm{CH}_{3} \mathrm{R}^{2}=\mathrm{H} \quad \mathrm{R}^{3}=\mathrm{Br}$
a: $R^{1}=H \quad R^{2}=H \quad R^{3}=H$
d: $\mathrm{R}^{1}=\mathrm{Cl} \quad \mathrm{R}^{2}=\mathrm{H} \quad \mathrm{R}^{3}=\mathrm{H}$
e: $\mathrm{R}^{1}=\mathrm{CH}_{3} \quad \mathrm{R}^{2}=\mathrm{H} \quad \mathrm{R}^{3}=\mathrm{H}$
f: $\mathrm{R}^{1}=\mathrm{Cl} \quad \mathrm{R}^{2}=\mathrm{CH}_{3} \mathrm{R}^{3}=\mathrm{H}$

Scheme 5. Synthesis of 2-(4-oxo-4H-chromen-2-yl)-3-(phenylamino)-2,3-dihydroquinazolin-4(1H)-ones (19a-f) and 3-(\{4-[(1H-1,2,4-triazol-1-yl)methyl]phenyl\}amino)-2-(4-oxo-4H-chromen-2-yl)-2,3dihydroquinazolin-4(1H)-one (20a-d)

\section{Biological Activity}

\section{In vitro antibacterial activity}

The hybrid molecules synthesized were tested against Gram-positive bacteria Staphylococcus aureus (MTCC 96), Streptococcus pyogenes (MTCC 442), and Gram-negative bacteria Escherichia coli (MTCC 443), and Pseudomonas aeruginosa (MTCC 741) (Table 1). Compounds $\mathbf{1 1 b}, \mathbf{1 9 a - c , 2 0 b}$ showed very good activity against the gram-positive organism Staphylococcus aureus compared to the standard drug Ampicillin. The compound 19b exhibited higher activity against gram-negative organism Escherichia coli when compared with the drug Ampicillin. 
Table 1. Antimicrobial activity results of the synthesized compounds

\begin{tabular}{cccccc}
\hline \multicolumn{5}{c}{ Minimal inhibition concentration $(\mathrm{\mu g} / \mathrm{ml})$} \\
\hline \multirow{2}{*}{ S. No } & Compound & $\begin{array}{c}\text { E.coli } \\
\text { MTCC 443 }\end{array}$ & $\begin{array}{c}\text { P. aeruginosa } \\
\text { MTCC 441 }\end{array}$ & $\begin{array}{c}\text { S. aureus } \\
\text { MTCC 96 }\end{array}$ & $\begin{array}{c}\text { S. pyogenus } \\
\text { MTCC 442 }\end{array}$ \\
\cline { 2 - 6 } & Ampicillin & 100 & 100 & 250 & 100 \\
1 & Ciprofloxacin & 25 & 25 & 50 & 50 \\
2 & 11a & 200 & 250 & 250 & 250 \\
3 & 11b & 500 & 125 & 62.5 & 125 \\
4 & 15a & 250 & 250 & 250 & 200 \\
5 & 19a & 500 & 500 & 125 & 250 \\
6 & 19b & 62.5 & 100 & 100 & 125 \\
7 & 19c & 100 & 125 & 125 & 125 \\
8 & 19e & 125 & 200 & 200 & 250 \\
9 & 19f & 250 & 125 & 250 & 200 \\
10 & 20a & 125 & 200 & 200 & 125 \\
11 & 20b & 500 & 200 & 100 & 200 \\
12 & 20c & 200 & 200 & 200 & 500 \\
\hline & 20d & 250 & 250 & 200 & 250 \\
\hline
\end{tabular}

\section{In vitro antifungal activity}

The in vitro antifungal activitiy of the synthesized compounds were tested against fungal strains, Candida albicans (MTCC 227), Aspergillus niger (MTCC 282) and Aspergillus clavatus (MTCC 1323) (Table 2). The compounds $15 \mathrm{a}, \mathbf{1 9} \mathrm{e}-\mathrm{f}, \mathbf{2 0}$ c showed more activity against Candida albicans when compared to the standard drug Griseofulvin.

Table-2. Antifungal activity results of the synthesized compounds

\begin{tabular}{ccccc}
\hline \multicolumn{5}{c}{ Minimal fungicidal concentration $(\mu \mathrm{g} / \mathrm{ml})$} \\
\hline \multirow{2}{*}{ S. No } & Compound & C. albicans & A. niger & A. clavatus \\
& MTCC 227 & MTCC 282 & MTCC 1323 \\
\hline Test Std. & Griseofulvin & 500 & 100 & 100 \\
1 & 11a & 500 & $>1000$ & $>1000$ \\
2 & 11b & 500 & 500 & 500 \\
3 & 15a & 250 & $>1000$ & $>1000$ \\
4 & 19a & 500 & 1000 & 1000 \\
5 & 19b & 1000 & $>1000$ & $>1000$ \\
6 & 19c & 1000 & $>1000$ & $>1000$ \\
7 & 19e & 250 & $>1000$ & $>1000$ \\
8 & 19f & 200 & $>1000$ & $>1000$ \\
9 & 20a & 1000 & $>1000$ & $>1000$ \\
10 & 20b & $>1000$ & 250 & 500 \\
11 & 20c & 250 & $>1000$ & $>1000$ \\
12 & 20d & $>1000$ & 250 & 250 \\
\hline
\end{tabular}




\section{Antimalarial activity}

The antimalarial activity of the synthesized compounds was evaluated and compared with standard drugs Chloroquine and quinine. Activity of all the synthesized compounds was found to be lower than the standard drugs.

\section{Conclusion}

In summary, we have developed a simple, efficient and convenient method for the synthesis of novel chromone / 2,3-dihydroquinazolin-4-one hybrid heterocycles by coupling of 2-aminobenzamide / 2-aminobenzohydrazides with chromone-2-carbaldehydes. Some of the synthesized compounds were found to exhibit moderate to very good antimicrobial activity. Hence, derivatives of chromone / 2,3-dihydroquinazolin-4-ones can be utilized in the future for the development of potent antimicrobial drugs.

\section{Experimental Section}

General. Electrospray ionization and tandem mass spectrometry experiments were performed using a triple quadrupole mass spectrometer (PE Sciex model API 3000). The positive and negative electrospray data were obtained by switching the capillary voltage between +5000 and $-4500 \mathrm{~V}$, respectively. For HRMS, UPLC-TOFMS system consisted of an Acquity ${ }^{\mathrm{TM}}$ Ultra Performance Liquid Chromatography system and Micromass LCT Premier XE Mass Spectrometer (High sensitivity orthogonal time-of-flight instrument; Waters, Milford, USA) equipped with an ESI lock spray source for accurate mass values. Leucine-enkephalin was used as reference compound, was introduced via the lock spray channel.

The NMR experiments were performed on Varian spectrometers operating at 400 and $500 \mathrm{MHz}$ in DMSO- $d_{6}$ at $30{ }^{\circ} \mathrm{C}$. The ${ }^{1} \mathrm{H}$ chemical shift values were reported on the $\delta$ scale in ppm, relative to TMS $(\delta=0.00)$ and the ${ }^{13} \mathrm{C}$ chemical shift values were reported relative to DMSO $(\delta=40 \mathrm{ppm})$ as internal standard. Standard pulse sequences provided by Varian were used for distortionless enhancement by polarization transfer (DEPT), gradient double quantum filtered correlation spectroscopy (gDQCOSY), and gradient heteronuclear single quantum coherence spectroscopy (gHSQC).

Biological activity measurements were performed at $\mathrm{M} / \mathrm{s}$ Microcare Laboratories, Surat, India. Antibacterial activity: Minimum inhibitory concentration (MIC) assay of the hybrid molecules synthesized was done by broth dilution method in tubes for macro dilution and in plates for micro dilution. Muller Hinton broth was used as nutrient medium to grow and dilute the drug suspension for the test bacteria. DMSO was used as diluent to get the desired concentration of synthesized compounds. Standard drugs ampicillin and ciprofloxacin were used for comparison.

Antifungal activity was performed against the fungal strains Candida albicans (MTCC 227), Aspergillus niger (MTCC 282) and Aspergillus clavatus (MTCC 1323). Fungal growth was done with Sabourauds dextrose broth at $28.8{ }^{\circ} \mathrm{C}$ in aerobic condition for $48 \mathrm{hrs} .2 \%$ DMSO and sterilized distilled water were used as negative control and Griseofulvin ( $1 \mathrm{U}$ strength) was used as positive control. Results were recorded in the form of primary and secondary screening.

Antimalarial Activity. The in vitro antimalarial assay was carried out in 96 well microtitre plates according to the micro assay protocol reference. Chloroquine and Quinine were taken as the reference drug for comparison. 


\section{General procedure for synthesis of 4-oxo-4H-chromen-2-carbaldehydes (9a-c)}

1,4-dioxane (10 volumes), 2-methyl-4H-chromene-4-ones (7a-c) $(160 \mathrm{mg}, 1.0 \mathrm{mmol})$ and a catalytic amount of hydrogen peroxide were placed in a three-necked flask and selenium dioxide (177.5 mg, $1.6 \mathrm{mmol}$ ) was added under stirring and heated to $100-105^{\circ} \mathrm{C}$. After completion of the reaction (16 h.; TLC monitoring), the reaction mass was cooled to $25-35^{\circ} \mathrm{C}$ and the selenium salts were removed by filtration. The filtrate was concentrated and the crude compound was purified by silica gel column chromatography. Elution of the column with ethyl acetate/petroleum ether 15:85 gave compounds $9 a-c$ in $60 \%$ yield.

\section{General procedure for synthesis of 4-oxo-4H-chromen-2-carbaldehydes (9d-f)}

Chlorobenzene (15 volumes), 2-(hydroxymethyl)-4H-chromene-4-one (8a-c) (176 mg, $1.0 \mathrm{mmol}$ ) and $\mathrm{MnO}_{2}$ (348 $\mathrm{mg}, 4.0 \mathrm{mmol}$ ) were placed in a three-necked flask and heated to $130-135^{\circ} \mathrm{C}$. After completion of the reaction (24 h. TLC monitoring), the reaction mass was cooled to $25-35{ }^{\circ} \mathrm{C}$ and the manganese salts were removed by filtration. The filtrate was concentrated and the crude compound was purified by silica gel column chromatography. Elution of column with ethyl acetate/petroleum ether 15:85 gave compounds $\mathbf{9 d - f}$ in $70 \%$ yield.

\section{General procedure for synthesis of 2-(4-oxo-4H-chromen-2-yl)-2,3-dihydroquinazolin-4(1H)-ones (11a-d)}

4-Oxo-4H-chromene-2-carbaldehyde $(9 \mathrm{a}-\mathrm{d})(1.0 \mathrm{mmol})$, ethanol (10 volumes) and PTSA (catalytic amount) were placed in a three-necked flask and 2-aminobenzamide $(1.2 \mathrm{mmol})$ was added under stirring and heated to $75-80^{\circ} \mathrm{C}$. After completion of the reaction ( $3 \mathrm{~h}$. TLC monitoring), the reaction mass was cooled to $20-25^{\circ} \mathrm{C}$ and filtered. The crude compound was purified by silica gel column chromatography. Elution of column with ethyl acetate/petroleum ether 20:80 gave compounds 11a-d.

2-(4-Oxo-4H-chromen-2-yl)-2,3-dihydroquinazolin-4(1H)-one (11a). Off-white crystalline solid (195 mg, 67\%). Mp 282-285 ${ }^{\circ} \mathrm{C}$. IR (solid, $\mathrm{KBr}, v_{\max }, \mathrm{cm}^{-1}$ ): 3318 ( $\mathrm{NH}$, sec amine), 1721 (C=O, ketone), 1672 (C=O, amide). ${ }^{1} \mathrm{H}$ NMR (400 MHz, DMSO- $d_{6}$ ): $\delta_{\mathrm{H}} 8.67$ (d, J $2.8 \mathrm{~Hz}, 1 \mathrm{H}, \mathrm{H}-3$ ', amide NH), 8.00 (dd, $J_{1} 7.60 \mathrm{~Hz}, J_{2} 0.8 \mathrm{~Hz}, 1 \mathrm{H}, \mathrm{H}-5$ ), $7.78\left(\mathrm{dt}, J_{1} 8.4 \mathrm{~Hz}, J_{2} 1.6 \mathrm{~Hz}, 1 \mathrm{H}, \mathrm{H}-7\right), 7.64\left(\mathrm{~d}, J 6.8 \mathrm{~Hz}, 1 \mathrm{H}, \mathrm{H}-5^{\prime}\right), 7.50\left(\mathrm{~m}, 3 \mathrm{H}, \mathrm{H}-6, \mathrm{H}-8\right.$ and $\left.1^{\prime} \mathrm{H}-\mathrm{NH}\right), 7.31$ (dt, $J_{1}$ $\left.8.4 \mathrm{~Hz}, J_{2} 1.6 \mathrm{~Hz}, 1 \mathrm{H}, \mathrm{H}-7^{\prime}\right), 6.83\left(\mathrm{~d}, J 8.0 \mathrm{~Hz}, 1 \mathrm{H}, \mathrm{H}-8^{\prime}\right), 6.72\left(\mathrm{t}, J 7.6 \mathrm{~Hz}, 1 \mathrm{H}, \mathrm{H}-6^{\prime}\right), 6.28(\mathrm{~s}, 1 \mathrm{H}, \mathrm{H}-3), 5.76$ (t, J 3.2 $\left.\mathrm{Hz}, 1 \mathrm{H}, \mathrm{H}-2^{\prime}\right) .{ }^{13} \mathrm{C}$ NMR (100 MHz, DMSO- $\left.d_{6}\right): \delta_{\mathrm{c}} 176.9$ (C-4, C=O), 166.9 (C-2), 162.9 (C-4', amide C=0), 155.6 (C-8a), 146.5 (C-8'a), 134.7 (C-7'), 133.7 (C aromatic), 127.4 (C aromatic), 125.7 (C aromatic), 124.9 (C-5), 123.1 (C aromatic), 118.3 (C aromatic), 117.8 (C-8), 114.7 (C aromatic), 114.5 (C aromatic), 108.1 (C-3) and 63.3 ppm (C-2'). ESI-MS m/z: 293.093. HRMS (ESI): $\mathrm{m} / z$ calcd for $\mathrm{C}_{17} \mathrm{H}_{13} \mathrm{~N}_{2} \mathrm{O}_{3}[\mathrm{M}+\mathrm{H}]^{+}:$: 293.0926; found: 293.0936.

2-(3-Bromo-4-oxo-4H-chromen-2-yl)-2,3-dihydroquinazoline-4(1H)-one (11b). Yellow crystalline solid (260 mg, 70\%). mp 235-238 ${ }^{\circ} \mathrm{C}$. IR (solid, $\mathrm{KBr}, v_{\max }, \mathrm{cm}^{-1}$ ): 3297 ( $\mathrm{NH}$, sec amine), 1740 (C=O, ketone), 1672 (C=O, amide). ${ }^{1} \mathrm{H}$ NMR (400 MHz, DMSO-d $)_{\text {) }} \delta_{\mathrm{H}} 8.44\left(\mathrm{~d}, J 1.6 \mathrm{~Hz}, 1 \mathrm{H}, \mathrm{H}-3\right.$ ', amide NH), 8.07 (dd, $J_{1} 6.8 \mathrm{~Hz}, J_{2} 1.2 \mathrm{~Hz}$, $1 \mathrm{H}, \mathrm{H}-5), 7.8(\mathrm{~m}, 1 \mathrm{H}, \mathrm{H}-7), 7.72\left(\mathrm{~d}, J 6.4 \mathrm{~Hz}, 1 \mathrm{H}, \mathrm{H}-5^{\prime}\right), 7.52(\mathrm{t}, J 6.4 \mathrm{~Hz}, 1 \mathrm{H}, \mathrm{H}-6), 7.42\left(\mathrm{~s}, 1 \mathrm{H}, \mathrm{H}-\mathrm{I}^{\prime}, \mathrm{NH}\right), 7.28$ (dt, $\left.J_{1} 6.4 \mathrm{~Hz}, J_{2} 1.2 \mathrm{~Hz}, 1 \mathrm{H}, \mathrm{H}-7^{\prime}\right), 7.16(\mathrm{~d}, J 6.8 \mathrm{~Hz}, 1 \mathrm{H}, \mathrm{H}-8), 6.76\left(\mathrm{t}, J 6.0 \mathrm{~Hz}, 1 \mathrm{H}, \mathrm{H}-6^{\prime}\right), 6.74\left(\mathrm{~d}, J 6.8 \mathrm{~Hz}, \mathrm{H}-8^{\prime}\right)$ and $6.27 \mathrm{ppm}\left(\mathrm{t}, J 2.4 \mathrm{~Hz}, 1 \mathrm{H}, \mathrm{H}-2^{\prime}\right) .{ }^{13} \mathrm{C}$ NMR (100 MHz, DMSO- $\left.d_{6}\right)$ : $\delta_{\mathrm{C}} 171.9$ (C-4, C=0), 165.7 (C-2), 161.9 (C-4', amide C=0), 152.9 (C-8a), 145.9 (C-8'a), 135.7 (C-7'), 134.9 (C aromatic), 128.4 (C aromatic), 125.7 (C aromatic), 124.7 (C-5), 123.1 (C aromatic), 118.1 (C aromatic), 117.8 (C-8), 114.8 (C aromatic), 114.5 (C aromatic), 108.1 (C-3) and $63.3 \mathrm{ppm}\left(\mathrm{C}-2^{\prime}\right)$. ESI-MS m/z: $371.0033[\mathrm{M}+\mathrm{H}]^{+}, \mathrm{m} / \mathrm{z} 373.0013[\mathrm{M}+2+\mathrm{H}]^{+}$. HRMS (ESI): $\mathrm{m} / \mathrm{z}$ calcd for $\mathrm{C}_{17} \mathrm{H}_{12} \mathrm{BrN}_{2} \mathrm{O}_{3}[\mathrm{M}+\mathrm{H}]^{+}: 371.0031$; found: 371.0033 .

2-(3-Bromo-6-methyl-4-oxo-4H-chromen-2-yl)-2,3-dihydroquinazolin-4(1H)-one (11c). Brown crystalline solid (270 mg, 70\%). mp 250-252 ${ }^{\circ} \mathrm{C}$. IR (solid, $\mathrm{KBr}, v_{\max }, \mathrm{cm}^{-1}$ ): 3317 ( $\mathrm{NH}, \mathrm{sec}$ amine), 1738 (C=O, ketone), 1672 (C=O, amide). ${ }^{1} \mathrm{H}$ NMR (400 MHz, DMSO- $\left.d_{6}\right): \delta_{\mathrm{H}} 8.43\left(\mathrm{~d}, J 2.0 \mathrm{~Hz}, 1 \mathrm{H}, \mathrm{H}-3^{\prime}\right.$, amide NH), $7.85(\mathrm{~d}, J 1.2 \mathrm{~Hz}, 1 \mathrm{H}, \mathrm{H}-$ 5), 7.71 (dd, J $J_{1} 6.0 \mathrm{~Hz}, J_{2} 1.2 \mathrm{~Hz}, 1 \mathrm{H}, \mathrm{H}-5^{\prime}$ ), 7.60 (dd, J $J_{1} 6.8 \mathrm{~Hz}, J_{2} 1.2 \mathrm{~Hz}, 1 \mathrm{H}, \mathrm{H}-7$ ), 7.41 (s, 1H, H-1'-NH), 7.27 (dt, 
$\left.J_{1} 6.8 \mathrm{~Hz}, J_{2} 1.2 \mathrm{~Hz}, 1 \mathrm{H}, \mathrm{H}-7^{\prime}\right), 7.07$ (d, J $6.8 \mathrm{~Hz}, 1 \mathrm{H}, \mathrm{H}-8$ ), 6.76 (t, J $\left.6.4 \mathrm{~Hz}, 1 \mathrm{H}, \mathrm{H}-6^{\prime}\right), 6.72$ (d, J $\left.6.4 \mathrm{~Hz}, 1 \mathrm{H}, \mathrm{H}-8^{\prime}\right)$, $6.26\left(\mathrm{t}, J 2.4 \mathrm{~Hz}, 1 \mathrm{H}, \mathrm{H}-2^{\prime}\right)$ and $2.36 \mathrm{ppm}(\mathrm{s}, 3 \mathrm{H}, \mathrm{H}-6 \mathrm{a}) .{ }^{13} \mathrm{C} \mathrm{NMR}\left(100 \mathrm{MHz}, \mathrm{DMSO}-d_{6}\right): \delta_{c} 172.1$ (C-4, C=O), 165.0 (C-2), 161.6 (C-4', amide C=0), 153.6 (C-8a), 144.9 (C-8'a), 135.0 (C-7'), 134.3 (C aromatic), 133.7 (C aromatic), 127.9 (C aromatic), 124.7 (C-5), 123.2 (C aromatic), 118.3 (C aromatic), 117.9 (C-8), 114.7 (C aromatic), 114.5 (C aromatic), 108.1 (C-3), 63.7 (C-2') and 20.6 ppm (C-6a). ESI-MS m/z: $385.0185[\mathrm{M}+\mathrm{H}]^{+}, \mathrm{m} / \mathrm{z} 387.018$ $[\mathrm{M}+2+\mathrm{H}]^{+}$. HRMS (ESI): $\mathrm{m} / z$ calcd for $\mathrm{C}_{18} \mathrm{H}_{14} \mathrm{BrN}_{2} \mathrm{O}_{3}[\mathrm{M}+\mathrm{H}]^{+}$: 385.0188; found: 385.0193 .

2-(6-Chloro-4-oxo-4H-chromen-2-yl)-2,3-dihydroquinazolin-4(1H)-one (11d). Yellow crystalline solid (180 mg, 55\%). mp 260-262 ${ }^{\circ} \mathrm{C}$. IR (solid, $\mathrm{KBr}, v_{\max }, \mathrm{cm}^{-1}$ ): 3296 ( $\mathrm{NH}$, sec amine), 1738 ( $\mathrm{C}=\mathrm{O}$, ketone), 1688 (C=O, amide). ${ }^{1} \mathrm{H}$ NMR (400 MHz, DMSO- $\left.d_{6}\right): \delta \mathrm{H} 8.69$ (d, J $2.8 \mathrm{~Hz}, 1 \mathrm{H}, \mathrm{H}-3$ ', amide NH), 7.93 (d, J $\left.2.8 \mathrm{~Hz}, 1 \mathrm{H}, \mathrm{H}-5\right), 7.85$ (dd, J1 $9.6 \mathrm{~Hz}, J_{2} 3.2 \mathrm{~Hz}, 1 \mathrm{H}, \mathrm{H}-5^{\prime}$ ), 7.63 (dd, J $\left.16.0 \mathrm{~Hz}, J_{2} 1.6 \mathrm{~Hz}, 1 \mathrm{H}, \mathrm{H}-7\right), 7.55$ (d, J $8.8 \mathrm{~Hz}, 1 \mathrm{H}, \mathrm{H}-8$ ), 7.51 (s, $1 \mathrm{H}, \mathrm{H}-1^{\prime}$ $\mathrm{NH}), 7.30\left(\mathrm{dt}, J_{1} 8.4 \mathrm{~Hz}, J_{2} 1.2 \mathrm{~Hz}, 1 \mathrm{H}, \mathrm{H}^{-7} \mathbf{7}^{\prime}\right), 6.83\left(\mathrm{~d}, J 7.6 \mathrm{~Hz}, 1 \mathrm{H}, \mathrm{H}-8^{\prime}\right), 6.73\left(\mathrm{t}, J 8.0 \mathrm{~Hz}, 1 \mathrm{H}, \mathrm{H}-6^{\prime}\right), 6.32(\mathrm{~s}, 1 \mathrm{H}, \mathrm{H}-$ 3) and $\delta_{H} 5.77 \mathrm{ppm}\left(\mathrm{t}, J 3.2 \mathrm{~Hz}, 1 \mathrm{H}, \mathrm{H}-2^{\prime}\right) .{ }^{13} \mathrm{C} \mathrm{NMR}\left(100 \mathrm{MHz}, \mathrm{DMSO}-d_{6}\right): \delta 175.8$ (C-4, C=O), 165.8 (C-2), 162.6 (C-4', amide C=O), 154.2 (C-8a), 147.5 (C-8'a), 134.6 (C-7'), 134.2 (C aromatic), 129.7 (C aromatic), 127.3 (C aromatic), 125.7 (C aromatic), 123.9 (C aromatic), 118.2 (C-8), 117.9 (C aromatic), 114.7 (C aromatic), 114.2 (C aromatic), 108.9 (C-3) and $63.8 \mathrm{ppm}\left(\mathrm{C}-2^{\prime}\right)$. ESI-MS m/z: $327.05[\mathrm{M}+\mathrm{H}]^{+}, \mathrm{m} / \mathrm{z} 329.04[\mathrm{M}+2+\mathrm{H}]^{+}$. HRMS (ESI): $\mathrm{m} / \mathrm{z}$ calcd for $\mathrm{C}_{17} \mathrm{H}_{12} \mathrm{ClN}_{2} \mathrm{O}_{3}[\mathrm{M}+\mathrm{H}]^{+}$; 327.0536; found: 327.0533 .

\section{Synthesis of 2-amino-N'-[(4-oxo-4H-chromen-2-yl)methylene]benzohydrazides (15a-f)}

4-Oxo-4H-chromene-2-carbaldehydes (9a-f) $(1.0 \mathrm{mmol})$, ethanol (10 volumes) were placed in a three-necked flask and 2-aminobenzohydrazide (14a) $(1.2 \mathrm{mmol})$ was added under stirring and heated to $75-80{ }^{\circ} \mathrm{C}$. After completion of the reaction ( $3 \mathrm{~h}$. TLC monitoring), the reaction mass was cooled to $20-25^{\circ} \mathrm{C}$ and the compound was filtered and dried at $70^{\circ} \mathrm{C}$ for $4 \mathrm{~h}$.

2-Amino-N'-[(4-oxo-4H-chromen-2-yl)methylene]benzohydrazide (15a). Yellow crystalline solid (245 mg, 80\%). mp 188-192 ${ }^{\circ} \mathrm{C}$. IR (solid, $\left.\mathrm{KBr}, v_{\max }, \mathrm{cm}^{-1}\right)$ : $3466(\mathrm{NH}$, primary amine), 3351 ( $\mathrm{NH}$, sec amine), 1738 (C=O, ketone), 1663 (C=O, amide). ${ }^{1} \mathrm{H}$ NMR (400 MHz, DMSO-d 6 ): $\delta_{\mathrm{H}} 12.16(\mathrm{~s}, 1 \mathrm{H}, \mathrm{H}-1$ 'b, amide NH), $8.24(\mathrm{~s}, 1 \mathrm{H}, \mathrm{H}-$ 2a), 8.06 (dd, J $\left.J_{1} 8.4 \mathrm{~Hz}, J_{2} 2.0 \mathrm{~Hz}, 1 \mathrm{H}, \mathrm{H}-5\right), 7.86$, (m, 1H, H-7), 7.73 (d, J $8.0 \mathrm{~Hz}, 1 \mathrm{H}, \mathrm{H}-8$ ), 7.62 (dd, J $8.0 \mathrm{~Hz}, J_{2}$ $\left.1.2 \mathrm{~Hz}, 1 \mathrm{H}, \mathrm{H}-2^{\prime}\right), 7.53$ (dt, $\left.J_{1} 7.6 \mathrm{~Hz}, J_{2} 0.8 \mathrm{~Hz}, 1 \mathrm{H}, \mathrm{H}-6\right), 7.26$ (dt, $\left.J_{1} 8.4 \mathrm{~Hz}, J_{2} 1.2 \mathrm{~Hz}, 1 \mathrm{H}, \mathrm{H}-4{ }^{\prime}\right), 6.78(\mathrm{~d}, J 8.0 \mathrm{~Hz}$, $\left.1 \mathrm{H}, \mathrm{H}-5^{\prime}\right), 6.72(\mathrm{~s}, 1 \mathrm{H}, \mathrm{H}-3), 6.61\left(\mathrm{dt}, J_{1} 8.4 \mathrm{~Hz}, J_{2} 0.8 \mathrm{~Hz}, 1 \mathrm{H}, \mathrm{H}-3^{\prime}\right)$ and $6.53 \mathrm{ppm}\left(\mathrm{s}, 2 \mathrm{H}, \mathrm{H}-6 \mathrm{a}^{\prime}, \mathrm{NH}_{2}\right) .{ }^{13} \mathrm{C} N M R$ (100 MHz, DMSO-d6): $\delta_{c} 176.8$ (C-4, C=0), 165.7 (C1'a, C=O amide), 159.2 (C-2), 155.5 (C-8a), 150.6 (C aromatic), 138.7 (C-2a), 134.7 (C-7), 132.9 (C4'), 128.5 (C-2'), 125.6 (C-6), 124.9 (C-5), 123.8 (C aromatic), 118.5 (C-8), 116.6 (C-5'), 114.6 (C-3'), 112.2 (C aromatic), and $111.7 \mathrm{ppm}(\mathrm{C}-3)$. ESI-MS m/z: 308.1 [M+H] ${ }^{+}$, HRMS (ESI): $m / z$ calcd for $\mathrm{C}_{17} \mathrm{H}_{14} \mathrm{~N}_{3} \mathrm{O}_{3}[\mathrm{M}+\mathrm{H}]^{+}$: 308.1035; found: 308.1046 .

2-Amino-N'-[(3-bromo-4-oxo-4H-chromen-2-yl)methylene]benzohydrazide (15b). Yellow crystalline solid (270 mg, 70\%). mp 254-257 ${ }^{\circ} \mathrm{C}$. IR (solid, $\mathrm{KBr}, v_{\max }, \mathrm{cm}^{-1}$ ): 3471 ( $\mathrm{NH}$, primary amine), 3357 ( $\mathrm{NH}$, sec amine), 1739 ( $\mathrm{C}=\mathrm{O}$, ketone), 1635 ( $\mathrm{C}=\mathrm{O}$, amide). ${ }^{1} \mathrm{H}$ NMR (400 MHz, DMSO- $\left.d_{6}\right): \delta_{\mathrm{H}} 12.4(\mathrm{~s}, 1 \mathrm{H}, \mathrm{H}-2 \mathrm{c}$, amide $\mathrm{NH}$ ), 8.87 (s, $1 \mathrm{H}, \mathrm{H}-2 \mathrm{a}), 8.00(\mathrm{~d}, 1 \mathrm{H}, J 8.0 \mathrm{~Hz}, \mathrm{H}-5), 7.90(\mathrm{t}, J 8.0 \mathrm{~Hz}, 1 \mathrm{H}, \mathrm{H}-7), 7.74(\mathrm{~d}, J 8.8 \mathrm{~Hz}, 1 \mathrm{H}, \mathrm{H}-8), 7.65(\mathrm{~d}, J 8.0 \mathrm{~Hz}$, $\left.1 \mathrm{H}, \mathrm{H}-2^{\prime}\right), 7.56(\mathrm{t}, J 7.6 \mathrm{~Hz}, 1 \mathrm{H}, \mathrm{H}-6), 7.26\left(\mathrm{t}, J 8.0 \mathrm{~Hz}, 1 \mathrm{H}, \mathrm{H}-4^{\prime}\right), 6.80\left(\mathrm{~d}, J 8.4 \mathrm{~Hz}, 1 \mathrm{H}, \mathrm{H}-5^{\prime}\right)$ and $6.60 \mathrm{ppm}(\mathrm{t}, J 7.6$

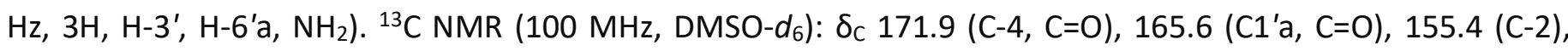
154.6 (C-8a), 150.8 (C aromatic), 138.0 (C-2a), 135.2 (C-7), 133.1 (C4'), 128.5 (C-2'), 126.0 (C-6), 125.4 (C-5), 121.6 (C aromatic), 118.3 (C-8), 116.6 (C-5'), 114.5 (C-3'), 111.9 (C aromatic) and 111.8 ppm (C-3). ESI-MS m/z: $386.01[\mathrm{M}+\mathrm{H}]^{+}, 388.01[\mathrm{M}+2+\mathrm{H}]^{+}$, HRMS (ESI): $\mathrm{m} / z$ calcd for $\mathrm{C}_{17} \mathrm{H}_{13} \mathrm{BrN}_{3} \mathrm{O}_{3}[\mathrm{M}+\mathrm{H}]^{+}$: 386.0140; found: 386.0139 .

2-Amino-N'-[(3-bromo-6-methyl-4-oxo-4H-chromen-2-yl)methylene]benzohydrazide (15c). Yellow crystalline solid (292 mg, 73\%). mp 236-239 ${ }^{\circ} \mathrm{C}$. IR (solid, $\left.\mathrm{KBr}, v_{\max }, \mathrm{cm}^{-1}\right): 3464(\mathrm{NH}$, primary amine), $3350(\mathrm{NH}$, sec amine), 1739 ( $\mathrm{C}=\mathrm{O}$, ketone), 1661 ( $\mathrm{C}=\mathrm{O}$, amide). ${ }^{1} \mathrm{H}$ NMR (400 MHz, CDCl 3 + DMSO-d 6 ): $\delta_{\mathrm{H}} 12.32$ (s, $1 \mathrm{H}, \mathrm{H}-1^{\prime} \mathrm{b}$, amide $\mathrm{NH}), 8.91(\mathrm{~s}, 1 \mathrm{H}, \mathrm{H}-2 \mathrm{a}), 7.91(\mathrm{~s}, 1 \mathrm{H}, \mathrm{H}-5), 7.64\left(\mathrm{~m}, 3 \mathrm{H}, \mathrm{H}-7, \mathrm{H}-8\right.$ and $\left.\mathrm{H}-2^{\prime}\right), 7.22\left(\mathrm{t}, 1 \mathrm{H}, \mathrm{H}-\mathrm{4}^{\prime}\right), 6.81(\mathrm{~d}, J 8.4$ 
$\left.\mathrm{Hz}, 1 \mathrm{H}, \mathrm{H}-5^{\prime}\right), 6.59\left(\mathrm{t}, 1 \mathrm{H}, \mathrm{H}-3^{\prime}\right) 6.50$ (s, 2H, H-6'a, NH 2 ) and $2.48 \mathrm{ppm}(\mathrm{s}, 3 \mathrm{H}, \mathrm{H}-6 \mathrm{a}) .{ }^{13} \mathrm{C}$ NMR (100 MHz, DMSO$\mathrm{d}_{6}$ ): $\delta_{c} 171.8$ (C-4, C=0), 165.9 (C1'a, C=0), 155.3 (C-2), 152.9 (C-8a), 150.7 (C aromatic), 138.1 (C-2a), 136.3 (C6), 135.8 (C-7), 133.1 (C-4'), 128.5 (C-2'), 124.6 (C-5), 121.4 (C aromatic), 118.1 (C-8), 116.6 (C-5'), 114.5 (C-3'), 111.8 (C aromatic), 111.8 (C-3) and 20.4 ppm (C-6a). ESI-MS m/z: $400.0[\mathrm{M}+\mathrm{H}]^{+}, 402.0[\mathrm{M}+2+\mathrm{H}]^{+}, \mathrm{HRMS}$ (ESI): $\mathrm{m} / z$ calcd for $\mathrm{C}_{18} \mathrm{H}_{15} \mathrm{BrN}_{3} \mathrm{O}_{3}[\mathrm{M}+\mathrm{H}]^{+}: 400.0297$; found: 400.0302 .

2-Amino-N'-[(6-Chloro-4-oxo-4H-chromen-2-yl)methylene]benzohydrazide (15d). Yellow crystalline solid (240 $\mathrm{mg}, 71 \%$ ). $\mathrm{mp} 218-220^{\circ} \mathrm{C}$. IR (solid, $\mathrm{KBr}, v_{\max }, \mathrm{cm}^{-1}$ ): 3459 ( $\mathrm{NH}$, primary amine), 3349 ( $\mathrm{NH}$, sec amine), 1742 ( $\mathrm{C}=\mathrm{O}$, ketone), 1673 ( $\mathrm{C}=\mathrm{O}$, amide). ${ }^{1} \mathrm{H}$ NMR (400 MHz, DMSO- $\left.d_{6}\right): \delta_{\mathrm{H}} 12.21(\mathrm{~s}, 1 \mathrm{H}, \mathrm{H}-1 \mathrm{l} \mathrm{b}$, amide $\mathrm{NH}$ ), 8.19 (s, $1 \mathrm{H}, \mathrm{H}-2 \mathrm{a}), 7.97$ (s, 1H, H-5), 7.82 (dt, J1 $\left.1.6 \mathrm{~Hz}, J_{2} 8.8 \mathrm{~Hz}, 1 \mathrm{H}, \mathrm{H}-7\right), 7.79$ (d, J $8.0 \mathrm{~Hz}, 1 \mathrm{H}, \mathrm{H}-8$ ), 7.61 (dd, J1 $1.6 \mathrm{~Hz}$, $\left.J_{2} 8.4 \mathrm{~Hz}, 1 \mathrm{H}, \mathrm{H}-2^{\prime}\right), 7.25$ (dt, J $\left.J_{1} 1.2 \mathrm{~Hz}, J_{2} 8.4 \mathrm{~Hz}, 1 \mathrm{H}, \mathrm{H}-4^{\prime}\right), 6.72\left(\mathrm{~d}, J 8.4 \mathrm{~Hz}, 1 \mathrm{H}, \mathrm{H}-5^{\prime}\right), 6.70(\mathrm{~s}, 1 \mathrm{H}, \mathrm{H}-3), 6.61$ (dt, $\left.J_{1} 1.2 \mathrm{~Hz}, J_{2} 8.4 \mathrm{~Hz}, 1 \mathrm{H}, \mathrm{H}-3^{\prime}\right)$ and $6.48 \mathrm{ppm}\left(\mathrm{s}, 2 \mathrm{H}, \mathrm{H}-6{ }^{\prime} \mathrm{a}, \mathrm{NH}_{2}\right) \cdot{ }^{13} \mathrm{C}$ NMR (100 MHz, DMSO-d $\left.)_{6}\right): \delta_{\mathrm{C}} 175.9$ (C-4, $\mathrm{C}=0$ ), 165.5 (C1'a, C=O amide), 160.2 (C-2), 155.5 (C-8a), 150.2 (C aromatic), 138.3 (C-2a), 134.1 (C-7), 133.1 (C-4'), 129.5 (C aromatic), 128.5 (C-2'), 125.6 (C aromatic), 123.9 (C aromatic), 118.3 (C-8), 116.8 (C-5'), 114.1 (C-3'), 112.5 (C aromatic), and $111.6 \mathrm{ppm}(\mathrm{C}-3)$. ESI-MS m/z: $342.1[\mathrm{M}+\mathrm{H}]^{+}, 344.1[\mathrm{M}+2+\mathrm{H}]^{+}$. HRMS (ESI): $\mathrm{m} / \mathrm{z}$ calcd for $\mathrm{C}_{17} \mathrm{H}_{13} \mathrm{ClN}_{3} \mathrm{O}_{3}[\mathrm{M}+\mathrm{H}]^{+}:$342.1049; found: 342.1055 .

2-Amino-N'-[(6-methyl-4-oxo-4H-chromen-2-yl)methylene]benzohydrazide (15e). Yellow crystalline solid (275 mg, 85\%). mp 249-251 ${ }^{\circ} \mathrm{C}$. IR (solid, $\mathrm{KBr}, v_{\max }, \mathrm{cm}^{-1}$ ): 3463 ( $\mathrm{NH}$, primary amine), 3345 ( $\mathrm{NH}$, sec amine), 1800 ( $\mathrm{C}=\mathrm{O}$, ketone), 1661 ( $\mathrm{C}=\mathrm{O}$, amide). ${ }^{1} \mathrm{H}$ NMR (400 MHz, DMSO-d 6 ): $\delta_{\mathrm{H}} 12.14(\mathrm{~s}, 1 \mathrm{H}, \mathrm{H}-1$ 'b, amide NH), 8.23 (s, 1H, H-2a), 7.84 (s, 1H, H-5), 7.65 (m, 3H), 7.26 (dt, J 8.4 Hz, J2 1.6 Hz, 1H, H-4'), 6.79 (d, J 7.6 Hz, 1H, H-5'), $6.67(\mathrm{~s}, 1 \mathrm{H}, \mathrm{H}-3) 6.61\left(\mathrm{t}, J 8.0 \mathrm{~Hz}, 1 \mathrm{H}, \mathrm{H}-3{ }^{\prime}\right), 6.51\left(\mathrm{~s}, 2 \mathrm{H}, \mathrm{H}-6 \mathrm{\prime}^{\mathrm{a}}, \mathrm{NH}_{2}\right)$ and $2.44 \mathrm{ppm}(\mathrm{s}, 3 \mathrm{H}, \mathrm{H}-6 \mathrm{a}) .{ }^{13} \mathrm{C}$ NMR (100 MHz, DMSO- $d_{6}$ ): $\delta_{c} 176.5$ (C-4, C=O), 165.8 (C1'a, C=O), 159.2 (C-2), 153.2 (C-8a), 150.5 (C aromatic), 138.3 (C2a), 136.3 (C-6), 135.8 (C-7), 132.8 (C-4'), 128.3 (C-2'), 124.1 (C-5), 123.9 (C aromatic), 118.1 (C-8), 116.5 (C-5'), 114.4 (C-3'), 111.9 (C aromatic), 111.7 (C-3) and 20.2 ppm (C-6a). ESI-MS m/z: $322.11[\mathrm{M}+\mathrm{H}]^{+}, \mathrm{HRMS}$ (ESI): $\mathrm{m} / \mathrm{z}$ calcd for $\mathrm{C}_{18} \mathrm{H}_{16} \mathrm{~N}_{3} \mathrm{O}_{3}[\mathrm{M}+\mathrm{H}]^{+}:$: 322.1192; found: 322.1184 .

2-Amino-N'-[(6-chloro-7-methyl-4-oxo-4H-chromen-2-yl)methylene]benzohydrazide (15f). Yellow crystalline solid (230 mg, 65\%). mp 246-248 ${ }^{\circ} \mathrm{C}$. IR (solid, $\left.\mathrm{KBr}, v_{\max }, \mathrm{cm}^{-1}\right): 3484(\mathrm{NH}$, primary amine), 3370 ( $\mathrm{NH}$, sec amine), 1673 (C=O, ketone), 1630 ( $\mathrm{C}=\mathrm{O}$, amide). ${ }^{1} \mathrm{H}$ NMR (400 MHz, DMSO- $\left.d_{6}\right): \delta_{\mathrm{H}} 12.16$ (s, $1 \mathrm{H}, \mathrm{H}-1$ 'b, amide $\mathrm{NH}), 8.22(\mathrm{~s}, 1 \mathrm{H}, \mathrm{H}-2 \mathrm{a}), 7.96(\mathrm{~s}, 1 \mathrm{H}, \mathrm{H}-5), 7.83(\mathrm{~s}, 1 \mathrm{H}, \mathrm{H}-8), 7.62\left(\mathrm{~d}, J 7.6 \mathrm{~Hz}, 1 \mathrm{H}, \mathrm{H}-2^{\prime}\right), 7.25$ (t, J $\left.7.2 \mathrm{~Hz}, 1 \mathrm{H}, \mathrm{H}-4^{\prime}\right)$, $6.78\left(\mathrm{~d}, J 8.4 \mathrm{~Hz}, 1 \mathrm{H}, \mathrm{H}-5^{\prime}\right), 6.72(\mathrm{~s}, 1 \mathrm{H}, \mathrm{H}-3), 6.59\left(\mathrm{t}, J 8.0 \mathrm{~Hz}, 1 \mathrm{H}, \mathrm{H}-3^{\prime}\right), 6.52\left(\mathrm{~s}, 2 \mathrm{H}, \mathrm{H}-6 \mathrm{\prime}^{\prime}, \mathrm{NH}_{2}\right)$ and $2.45 \mathrm{ppm}(\mathrm{s}$, $3 \mathrm{H}, \mathrm{H}-7 \mathrm{a}) .{ }^{13} \mathrm{C}$ NMR (100 MHz, DMSO- $\left.d_{6}\right): \delta_{\mathrm{C}} 175.6$ (C-4, C=O), 165.6 (C1'a, C=0), 159.3 (C-2), 154.0 (C aromatic), 150.6 (C aromatic), 143.1 (C aromatic), 138.5 (C-2a), 132.9 (C4'), 130.8 (C-6), 128.5 (C-2'), 124.1 (C5), 123.1 (C aromatic), 120.8 (C aromatic), 116.5 (C-5'), 114.5 (C-3'), 112.1 (C aromatic), 111.4 (C-3) and 20.1 ppm (C-6a). ESI-MS m/z: $356.07[\mathrm{M}+\mathrm{H}]^{+}, \mathrm{m} / z$ 358.07 [M+2+H] ${ }^{+}$, HRMS (ESI): $\mathrm{m} / z$ calcd for $\mathrm{C}_{18} \mathrm{H}_{15} \mathrm{ClN}_{3} \mathrm{O}_{3}[\mathrm{M}+\mathrm{H}]^{+}$: 356.0802; found: 356.0797.

\section{Synthesis of 3-\{[(4-oxo-4H-chromen-2-yl)methylene]amino\}-2-phenyl-2,3-dihydroquinazolin-4(1H)-one (18).}

2-Amino-N'-[(4-oxo-4H-chromen-2-yl)methylene]benzohydrazide (15a) (307 mg, $1.0 \mathrm{mmol}$ ), ethanol (10 volumes) were placed in a three-necked flask and benzaldehyde (127 mg, $1.2 \mathrm{mmol}$ ) was added under stirring and heated to $75-80^{\circ} \mathrm{C}$. After completion of reaction ( $3 \mathrm{~h}$. TLC monitoring), reaction mass was cooled to 20-25 ${ }^{\circ} \mathrm{C}$ and compound was filtered and dried at $70^{\circ} \mathrm{C}$ for $4 \mathrm{~h}$. to obtain a yellow crystalline solid ( $325 \mathrm{mg}, 82 \%$ ). $\mathrm{mp}$ 285-288 ${ }^{\circ} \mathrm{C}$. IR (solid, $\mathrm{KBr}, v_{\max }, \mathrm{cm}^{-1}$ ): 3298 ( $\mathrm{NH}$, sec amine), 1740 ( $\mathrm{C}=\mathrm{O}$, ketone), 1630 (C=O, amide). ${ }^{1} \mathrm{H}$ NMR (400 MHz, DMSO- $d_{6}$ ): $\delta_{\mathrm{H}} 8.81(\mathrm{~s}, 1 \mathrm{H}, \mathrm{H}-2 \mathrm{a}), 8.14\left(\mathrm{~d}, J 3.2 \mathrm{~Hz}, 1 \mathrm{H}, \mathrm{NH}\right.$ ), 8.04 (dd, J1 $8.0 \mathrm{~Hz}, J_{2} 1.2 \mathrm{~Hz}, 1 \mathrm{H}, \mathrm{H}-5$ ), $7.81\left(\mathrm{dt}, J_{1} 8.8 \mathrm{~Hz}, J_{2} 1.6 \mathrm{~Hz}, 1 \mathrm{H}, \mathrm{H}-7\right), 7.74(\mathrm{~d}, J 7.2 \mathrm{~Hz}, 1 \mathrm{H}, \mathrm{CH}$ aromatic), 7.68 (d, J $8.8 \mathrm{~Hz}, 1 \mathrm{H}, \mathrm{H}-8), 7.50$ (t, J 8.0 $\mathrm{Hz}, 1 \mathrm{H}, \mathrm{H}-6), 7.4-7.3(\mathrm{~m}, 6 \mathrm{H}, \mathrm{CH}$ aromatic), $6.83(\mathrm{~d}, J 8.0 \mathrm{~Hz}, 1-\mathrm{H}, \mathrm{CH}$ aromatic), 6.76 (t, J $7.6 \mathrm{~Hz}, 1 \mathrm{H}, \mathrm{CH}$ aromatic), $6.70(\mathrm{~s}, 1 \mathrm{H}, \mathrm{H}-3)$ and $6.67 \mathrm{ppm}\left(\mathrm{d}, J 2.8 \mathrm{~Hz}, 1 \mathrm{H}, \mathrm{H}-2^{\prime}\right) .{ }^{13} \mathrm{C}$ NMR (100 MHz, DMSO-d $)$ : $\delta_{c} 178.2$ (C-4, 
C=0), 162.5 (C-1'a, C=0), 159.9 (C-2), 156.1 (C-8a), 145.1 (C aromatic), 143.7 (C aromatic), 139.1 (C-2a), 134.9 (C-7), 134.0 (C-4'), 129.3 (C aromatic), 129.1 (C aromatic), 128.8 (C-3' and C-5'), 126.6 (C-2' and C-6'), 125.7 (C6), 125.0 (C aromatic), 124.5 (C-5), 120.2 (C-8), 118.3 (C-5'), 116.2 (C-3'), 115.2 (C aromatic), 110.9 (C-3) and $76.4 \mathrm{ppm}\left(\mathrm{C}-2^{\prime}\right)$. ESI-MS m/z: $396.2[\mathrm{M}+\mathrm{H}]^{+}$.

\section{Synthesis of 2-(4-oxo-4H-chromen-2-yl)-3-(phenylamino)-2,3-dihydroquinazolin-4(1H)-ones (19a-f)}

4-Oxo-4H-chromene-2-carbaldehyde $(9 \mathrm{a}-\mathrm{f})(1.0 \mathrm{mmol})$, and ethanol (10 volumes) were placed in a threenecked flask and 2-amino- $\mathrm{N}$--phenylbenzohydrazide (14b) $(1.2 \mathrm{mmol})$ was added under stirring and heated to $75-80^{\circ} \mathrm{C}$. After completion of the reaction ( $3 \mathrm{~h}$. TLC monitoring), the reaction mass was cooled to $20-25^{\circ} \mathrm{C}$ and filtered. The crude compound was purified by silica gel column chromatography. Elution of the column with ethyl acetate/petroleum ether 20:80 gave compounds 19a-f.

2-(4-Oxo-4H-chromen-2-yl)-3-(phenylamino)-2,3-dihydroquinazolin-4(1H)-one (19a). Off white crystalline solid (306 mg, 80\%). mp 153-155 ${ }^{\circ} \mathrm{C}$. IR (solid, $\mathrm{KBr}, v_{\max }, \mathrm{cm}^{-1}$ ): 3279 ( $\mathrm{NH}$, sec amine), 1649 (C=O, ketone), 1607 (C=O amide). ${ }^{1} \mathrm{H}$ NMR (400 MHz, DMSO- $\left.d_{6}\right): \delta_{\mathrm{H}} 8.61$ (s, $1 \mathrm{H}, \mathrm{H}-3 \mathrm{\prime}$ a), 8.00 (dd, $\left.J_{1} 8.0 \mathrm{~Hz}, J_{2} 1.6 \mathrm{~Hz}, 1 \mathrm{H}, \mathrm{H}-5\right), 7.88$ (d, J $\left.3.2 \mathrm{~Hz}, 1 \mathrm{H}, \mathrm{H}-1^{\prime}, \mathrm{NH}\right), 7.79(\mathrm{~m}, 1 \mathrm{H}, \mathrm{H}-7), 7.70$ (dd, J $\left.J_{1} 8.4 \mathrm{~Hz}, J_{2} 1.2 \mathrm{~Hz}, 1 \mathrm{H}, \mathrm{H}-5^{\prime}\right), 7.47$ (dt, J1 $8.0 \mathrm{~Hz}, J_{2} 1.2 \mathrm{~Hz}$, $1 \mathrm{H}, \mathrm{H}-6), 7.42$ (d, J $8.4 \mathrm{~Hz}, 1 \mathrm{H}, \mathrm{H}-8$ ), 7.34 (dt, J1 $\left.8.4 \mathrm{~Hz}, J_{2} 1.6 \mathrm{~Hz}, 1 \mathrm{H}, \mathrm{H}-7^{\prime}\right), 7.19$ (t, J $8.4 \mathrm{~Hz}, 2 \mathrm{H}, \mathrm{H}-3^{\prime}, \mathrm{H}_{-5}$ '), 6.8 (m, $5 \mathrm{H}, \mathrm{CH}$ aromatic), $6.42(\mathrm{~s}, 1 \mathrm{H}, \mathrm{H}-3)$ and $5.95 \mathrm{ppm}\left(\mathrm{d}, J 3.2 \mathrm{~Hz}, 1 \mathrm{H}, \mathrm{H}-2^{\prime}\right) .{ }^{13} \mathrm{C}$ NMR $\left(100 \mathrm{MHz}, \mathrm{DMSO}-d_{6}\right): \delta_{c}$ 176.9 (C-4, C=0), 165.3 (C-2), 162.5 (C-4', C=0), 155.6 (C-8a), 147.6 (C-8'a), 146.3 (C aromatic), 134.5 (C aromatic), 134.0 (C aromatic), 128.9 (C-3', 5'), 127.5 (C aromatic), 125.7 (C aromatic), 124.9 (C-5), 123.2 (C aromatic), 119.4 (C aromatic), 118.4 (C-8), 118.2 (C aromatic), 114.7 (C aromatic), 114.3 (C aromatic), 112.3 (C$2^{\prime}$ and $\left.C^{\prime}-6^{\prime}\right), 108.9$ (C-3) and 71.6 ppm (C-2'). ESI-MS m/z: $384.13[\mathrm{M}+\mathrm{H}]^{+}, \mathrm{HRMS}(\mathrm{ESI}): \mathrm{m} / z$ calcd for $\mathrm{C}_{23} \mathrm{H}_{18} \mathrm{~N}_{3} \mathrm{O}_{3}$ $[\mathrm{M}+\mathrm{H}]^{+}:$384.1348; found: 384.1333 .

2-(3-Bromo-4-oxo-4H-chromen-2-yl)-3-(phenylamino)-2,3-dihydroquinazolin-4(1H)-one (19b). Off white crystalline solid (322 mg, 70\%). mp 259-262 ${ }^{\circ} \mathrm{C}$. IR (solid, $\mathrm{KBr}, v_{\max }, \mathrm{cm}^{-1}$ ): 3300 ( $\mathrm{NH}$, sec amine), 1678 (C=O, ketone), 1664 (C=O, amide). ${ }^{1} \mathrm{H}$ NMR (400 MHz, DMSO-d 6 ): $\delta_{\mathrm{H}} 8.33$ (s, 1H, H-3'a, NH), 8.06 (dd, J $J_{1} 8.4 \mathrm{~Hz}, J_{2} 1.6$ $\mathrm{Hz}, 1 \mathrm{H}, \mathrm{H}-5), 7.85$ (d, J $\left.2.8 \mathrm{~Hz}, 1 \mathrm{H}, \mathrm{H}-1^{\prime}, \mathrm{NH}\right), 7.78\left(\mathrm{~m}, 2 \mathrm{H}, \mathrm{H}-7, \mathrm{H}-5^{\prime}\right), 7.52(\mathrm{t}, J 8.0 \mathrm{~Hz}, 1 \mathrm{H}, \mathrm{H}-6), 7.33(\mathrm{t}, J 6.8 \mathrm{~Hz}$, $\left.1 \mathrm{H}, \mathrm{H}-7^{\prime}\right), 7.17$ (t, J $\left.8.0 \mathrm{~Hz}, 2 \mathrm{H}, \mathrm{H}-3^{\prime}, \mathrm{H}_{-5} 5^{\prime}\right), 7.07$ (d, J $\left.8.4 \mathrm{~Hz}, 1 \mathrm{H}, \mathrm{H}-8\right), 6.80$ (m, 5H, CH aromatic) and $6.41 \mathrm{ppm}$ (d, J $\left.2.8 \mathrm{~Hz}, 1 \mathrm{H}, \mathrm{H}-2^{\prime}\right) .{ }^{13} \mathrm{C}$ NMR (100 MHz, DMSO-d 6 ): $\delta_{\mathrm{c}} 171.6$ (C-4, C=0), 163.0 (C-2) 161.5 (C-4', C=0), 154.3 (C-8a), 147.2 (C-8'a), 145.9 (C aromatic), 135.1 (C aromatic), 133.9 (C-7'), 129.0 (C-3', 5'), 127.3 (C-7), 126.3 (C aromatic), 125.5 (C aromatic), 121.2 (C aromatic), 119.5 (C aromatic), 118.1 (C-8), 117.9 (C aromatic), 114.3 (C aromatic), 113.9 (C aromatic), 112.3 (C-2" and C-6"), 107.3 (C-3) and 71.9 ppm (C-2'). ESI-MS m/z: 462.04 $[\mathrm{M}+\mathrm{H}]^{+}, m / z 464.04[\mathrm{M}+2+\mathrm{H}]^{+}, \mathrm{HRMS}(\mathrm{ESI}): \mathrm{m} / z$ calcd for $\mathrm{C}_{23} \mathrm{H}_{17} \mathrm{~N}_{3} \mathrm{O}_{3} \mathrm{Br}[\mathrm{M}+\mathrm{H}]^{+}:$462.0453; found: 462.0432 .

\section{2-(3-Bromo-6-methyl-4-oxo-4H-chromen-2-yl)-3-(phenylamino)-2,3-dihydroquinazolin-4(1H)-one}

(19c).

Yellow crystalline solid (284 mg, 60\%). mp 225-227 ${ }^{\circ} \mathrm{C}$. IR (solid, $\mathrm{KBr}, v_{\max }, \mathrm{cm}^{-1}$ ): 3372 (NH, sec amine), 1683 ( $\mathrm{C}=\mathrm{O}$, ketone), 1639 ( $\mathrm{C}=\mathrm{O}$, amide). ${ }^{1} \mathrm{H}$ NMR (400 MHz, DMSO- $\left.d_{6}\right) \delta_{\mathrm{H}} 8.34(\mathrm{~s}, 1 \mathrm{H}, \mathrm{H}-3$ 'a, NH), 7.83 (s, 2H, H-5, H1', NH), 7.75 (d, J $7.6 \mathrm{~Hz}, 1 \mathrm{H}, \mathrm{H}-7), 7.62(\mathrm{~d}, J 8.4 \mathrm{~Hz}, 1 \mathrm{H}, \mathrm{H}-5), 7.33\left(\mathrm{t}, J 7.2 \mathrm{~Hz}, 1 \mathrm{H}, \mathrm{H}-7^{\prime}\right), 7.15(\mathrm{t}, J 7.6 \mathrm{~Hz}, 2 \mathrm{H}, \mathrm{H}-$ 3', H-5'), 6.97 (d, J $8.4 \mathrm{~Hz}, 1 \mathrm{H}, \mathrm{H}-8), 6.80\left(\mathrm{~m}, 5 \mathrm{H}, \mathrm{CH}\right.$ aromatic), $6.38\left(\mathrm{~d}, J 2.8 \mathrm{~Hz}, 1 \mathrm{H}, \mathrm{H}-2^{\prime}\right)$ and $2.41 \mathrm{ppm}(\mathrm{s}, 3 \mathrm{H}$, H-6a). ${ }^{13} \mathrm{C}$ NMR (100 Hz, DMSO-d 6 ): $\delta_{c} 171.5$ (C-4, C=O), 163.0 (C-2), 161.3 (C=O, C-4', amide), 152.6 (C-8a), 147.2 (C-8'a), 145.9 (C aromatic), 136.2 (C-6), 136.0 (C aromatic), 133.9 (C aromatic), 128.9 (C-3', 5'), 127.3 (C aromatic), 124.6 (C aromatic), 121.0 (C aromatic), 119.5 (C aromatic), 118.1 (C-8), 117.8 (C aromatic), 114.3 (C aromatic), 113.9 (C aromatic), 112.3 (C-2' and C-6'), 107.2 (C-3), 71.9 (C-2') and 20.4 ppm (C-6a). ESI-MS m/z: $476.05[\mathrm{M}+\mathrm{H}]^{+}, 478.05[\mathrm{M}+2+\mathrm{H}]^{+}, \mathrm{HRMS}(\mathrm{ESI}): \mathrm{m} / z$ calcd for $\mathrm{C}_{24} \mathrm{H}_{19} \mathrm{~N}_{3} \mathrm{O} 33 \mathrm{Br}[\mathrm{M}+\mathrm{H}]^{+}:$476.0610; found: 476.0591. 2-(6-Chloro-4-oxo-4H-chromen-2-yl)-3-(phenylamino)-2,3-dihydroquinazolin-4(1H)-one (19d). Yellow crystalline solid (258 mg, 62\%). mp 198-200 ${ }^{\circ} \mathrm{C}$. IR (solid, KBr, $v_{\max }, \mathrm{cm}^{-1}$ ): 3289 ( $\mathrm{NH}$, sec amine), 1645 (C=O, ketone), 1602 (C=O, amide). ${ }^{1} \mathrm{H}$ NMR (400 MHz, DMSO- $\left.d_{6}\right)$ : $\delta_{\mathrm{H}} 8.59$ (s, $\left.1 \mathrm{H}, \mathrm{H}-3{ }^{\prime} \mathrm{a}\right), 7.89$ (d, J $\left.2.8 \mathrm{~Hz}, 1 \mathrm{H}, \mathrm{H}-5\right)$, 
7.85 (d, J $\left.2.8 \mathrm{~Hz}, 1 \mathrm{H}, \mathrm{H}-1^{\prime}, \mathrm{NH}\right), 7.79$ (t, J $6.0 \mathrm{~Hz}, 1.6 \mathrm{~Hz}, 1 \mathrm{H}, \mathrm{H}-7$ ), 7.72 (dd, J1 $8.4 \mathrm{~Hz}, J_{2} 1.2 \mathrm{~Hz}, 1 \mathrm{H}, \mathrm{H}-5$ '), 7.55 (d, J $8.4 \mathrm{~Hz}, 1 \mathrm{H}, \mathrm{H}-8), 7.31\left(\mathrm{dt}, J_{1} 8.4 \mathrm{~Hz}, J_{2} 1.6 \mathrm{~Hz}, 1 \mathrm{H}, \mathrm{H}-7^{\prime}\right), 7.10\left(\mathrm{t}, J 8.4 \mathrm{~Hz}, 2 \mathrm{H}, \mathrm{H}-3^{\prime}, \mathrm{H}-5^{\prime}\right), 6.77(\mathrm{~m}, 5 \mathrm{H}, \mathrm{CH}$ aromatic), $6.44(\mathrm{~s}, 1 \mathrm{H}, \mathrm{H}-3)$ and $5.91 \mathrm{ppm}\left(\mathrm{d}, J 3.2 \mathrm{~Hz}, 1 \mathrm{H}, \mathrm{H}-2^{\prime}\right) .{ }^{13} \mathrm{C}$ NMR (100 MHz, DMSO-d $\left.d_{6}\right): \delta_{\mathrm{c}} 175.9$ (C-4, C=0), 164.9 (C-2), 162.2 (C-4', C=O), 155.1 (C-8a), 147.0 (C-8'a), 146.1 (C aromatic), 134.5 (C aromatic), 134.0 (C aromatic), 129.7 (C aromatic), 128.9 (C-3', 5'), 127.6 (C aromatic), 124.9 (C-5), 123.2 (C aromatic), 119.4 (C aromatic), 118.4 (C-8), 118.3 (C aromatic), 114.8 (C aromatic), 114.3 (C aromatic), 112.3 (C-2' and C-6'), 108.8 (C-3) and $71.6 \mathrm{ppm}\left(\mathrm{C}-2^{\prime}\right)$. ESI-MS m/z: $418.08[\mathrm{M}+\mathrm{H}]^{+}, 420.08[\mathrm{M}+2+\mathrm{H}]^{+}, \mathrm{HRMS}(\mathrm{ESI}): \mathrm{m} / \mathrm{z}$ calcd for $\mathrm{C}_{23} \mathrm{H}_{17} \mathrm{ClN}_{3} \mathrm{O}_{3}[\mathrm{M}+\mathrm{H}]^{+}:$: 418.0912; found: 418.0812 .

2-(6-Methyl-4-oxo-4H-chromen-2-yl)-3-(phenylamino)-2,3-dihydroquinazolin-4(1H)-one (19e). Pale yellow crystalline solid (278 mg, 70\%). mp 213- $215{ }^{\circ} \mathrm{C}$. IR (solid, KBr, $v_{\max }, \mathrm{cm}^{-1}$ ): 3329 ( $\mathrm{NH}$, sec amine), 1653 (C=O, ketone), 1603 (C=O, amide). ${ }^{1} \mathrm{H}$ NMR (400 MHz, DMSO- $\left.d_{6}\right): \delta_{\mathrm{H}} 8.57\left(\mathrm{~s}, 1 \mathrm{H}, \mathrm{H}-3\right.$ 'a, NH), 7.84 (d, J 3.2 Hz, 1H, $1^{\prime}-\mathrm{H}_{\text {, }}$ $\mathrm{NH}), 7.77$ (d, J $1.2 \mathrm{~Hz}, 1 \mathrm{H}, \mathrm{H}-5), 7.70$ (d, J $8.0 \mathrm{~Hz}, 1 \mathrm{H}, \mathrm{H} 7), 7.61$ (dd, J $\left.8.0 \mathrm{~Hz}, J_{2} 2.0 \mathrm{~Hz}, 1 \mathrm{H}, \mathrm{H}-5^{\prime}\right), 7.33(\mathrm{~m}, 2 \mathrm{H}, \mathrm{H}-$ 8, H-7'), 7.19 (t, J $\left.8.0 \mathrm{~Hz}, 2 \mathrm{H}, \mathrm{H}-3^{\prime}, \mathrm{H}-5^{\prime}\right), 6.85$ (m, 5H, CH aromatic), $6.38(\mathrm{~s}, 1 \mathrm{H}, \mathrm{H}-3), 5.93$ (d, J $\left.2.8 \mathrm{~Hz}, 1 \mathrm{H}, \mathrm{H}-2^{\prime}\right)$ and $2.44 \mathrm{ppm}(\mathrm{s}, 3 \mathrm{H}, \mathrm{H}-6 \mathrm{a}) .{ }^{13} \mathrm{C}$ NMR (100 MHz, DMSO- $d_{6}$ ): $\delta_{\mathrm{C}} 177.3$ (C-4, C=0), 165.7 (C-2), 163.0 (C-3'a, amide C=O), 154.4 (C-8a), 148.1 (C aromatic), 146.0 (C aromatic), 136.0 (C-6), 135.9 (C aromatic), 133.8 (C-7'), 128.7 (C-3' and C-5'), 127.3 (C-7), 124.0 (C-5), 123.4 (C aromatic), 119.2 (C aromatic), 118.0 (C-8), 118.0 (C aromatic), 114.8 (C aromatic), 114.5 (C aromatic), 112.0 (C-2' and C-6'), 108.4 (C-3), 71.4 (C-2') and 20.2 ppm (C-6a). ESIMS $m / z: 398.14[\mathrm{M}+\mathrm{H}]^{+}$, HRMS (ESI): $\mathrm{m} / z$ calcd for $\mathrm{C}_{24} \mathrm{H}_{20} \mathrm{~N}_{3} \mathrm{O}_{3}[\mathrm{M}+\mathrm{H}]^{+}: 398.1505$; found: 398.1490 .

2-(6-Chloro-7-methyl-4-oxo-4H-chromen-2-yl)-3-(phenylamino)-2,3-dihydroquinazolin-4(1H)-one (19f). Pale yellow crystalline solid (327 mg, 76\%). mp 276-278 ${ }^{\circ} \mathrm{C}$. IR (solid, $\mathrm{KBr}, v_{\max }, \mathrm{cm}^{-1}$ ): 3285 ( $\mathrm{NH}$, sec amine), 1645 ( $\mathrm{C}=\mathrm{O}$, ketone), 1604 ( $\mathrm{C}=\mathrm{O}$, amide). ${ }^{1} \mathrm{H}$ NMR (400 MHz, DMSO- $\left.d_{6}\right): \delta_{\mathrm{H}} 8.56(\mathrm{~s}, 1 \mathrm{H}, \mathrm{H}-3$ 'a, NH), $7.91(\mathrm{~s}, 1 \mathrm{H}, \mathrm{H} 5)$, $7.84\left(\mathrm{~d}, J 3.2 \mathrm{~Hz}, 1 \mathrm{H}, \mathrm{H}-1^{\prime}, \mathrm{NH}\right), 7.70$ (d, J $\left.7.2 \mathrm{~Hz}, 1 \mathrm{H}, \mathrm{H}-5^{\prime}\right), 7.48(\mathrm{~s}, 1 \mathrm{H}, \mathrm{H}-8), 7.34\left(\mathrm{t}, J 8.4 \mathrm{~Hz}, 1 \mathrm{H}, \mathrm{H}-7^{\prime}\right), 7.19$ (t, $\left.8.0 \mathrm{~Hz}, 2 \mathrm{H}, \mathrm{H}-3^{\prime}, \mathrm{H}-5^{\prime}\right), 6.80(\mathrm{~m}, 5 \mathrm{H}), 6.43(\mathrm{~s}, 1 \mathrm{H}, \mathrm{H}-3), 5.96\left(\mathrm{~d}, \mathrm{~J} 2.8 \mathrm{~Hz}, 1 \mathrm{H}, \mathrm{H}-2^{\prime}\right)$ and $2.44 \mathrm{ppm}(\mathrm{s}, 3 \mathrm{H}, \mathrm{H}-7 \mathrm{a}) .{ }^{13} \mathrm{C}$ NMR (100 MHz, DMSO- $d_{6}$ ): $\delta c 177.3$ (C-4, C=0), 165.7 (C-2), 162.9 (C-3'a, amide C=0), 154.4 (C-8a), 148.2 (C8'a), 146.8 (C aromatic), 136.2 (C aromatic), 135.9 (C aromatic), 133.9 (C-7'), 130.5 (C aromatic), 128.8 (C-3' and $\mathrm{C}-5^{\prime}$ ), 127.4 (C aromatic), 124.0 (C-5), 119.9 (C aromatic), 119.3 (C aromatic), 118.0 (C-8), 117.9 (C aromatic), 114.5 (C aromatic), 112.0 (C-2' and C-6'), 109.2 (C-3), 72.3 (C-2') and 21.0 ppm (C-6a). ESI-MS m/z: $432.11[\mathrm{M}+\mathrm{H}]^{+}, 434.10[\mathrm{M}+2+\mathrm{H}]^{+}$, $\mathrm{HRMS}(\mathrm{ESI}): \mathrm{m} / z$ calcd for $\mathrm{C}_{24} \mathrm{H}_{19} \mathrm{ClN}_{3} \mathrm{O}_{3}[\mathrm{M}+\mathrm{H}]^{+}:$:32.1115; found: 432.1102.

Synthesis of 3-(\{4-[(1H-1,2,4-triazol-1-yl)methyl]phenyl\}amino)-2-(4-oxo-4H-chromen-2-yl)-2,3-dihydroquinazolin-4(1H)-ones (20a-d)

4-Oxo-4H-chromene-2-carbaldehyde $(9 a-d)(1.0 \mathrm{mmol})$, ethanol (10 volumes) were placed in a three-necked flask and $\mathrm{N}^{\prime}$-\{4-[(1H-1,2,4-triazol-1-yl)methyl]phenyl\}-2-aminobenzohydrazide (14c) (1.2 mmol) was added under stirring and heated to $75-80^{\circ} \mathrm{C}$. After completion of the reaction ( $3 \mathrm{~h}$. TLC monitoring), the reaction mass was cooled to $20-25{ }^{\circ} \mathrm{C}$ and filtered. The crude compound was purified by silica gel column chromatography. Elution of the column with ethyl acetate/petroleum ether 20:80 gave compounds 20a-d.

3-(\{4-[(1H-1,2,4-Triazol-1-yl)methyl]phenyl\}amino)-2-(4-oxo-4H-chromen-2-yl)-2,3-dihydroquinazolin-4(1H)one (20a). Pale yellow crystalline solid (255 mg, 55\%). mp 160-163 ${ }^{\circ} \mathrm{C}$. IR (solid, $\mathrm{KBr}, v_{\max }, \mathrm{cm}^{-1}$ ): 3259 (NH, sec amine), 1741 ( $\mathrm{C}=\mathrm{O}$, ketone), 1651 ( $\mathrm{C}=\mathrm{O}$, amide). ${ }^{1} \mathrm{H}$ NMR (400 MHz, DMSO- $\left.d_{6}\right): \delta_{\mathrm{H}} 8.66$ (s, 1H, H-3'a), 8.57 (s, $\left.1 \mathrm{H}, \mathrm{H}-5^{\prime \prime}\right), 7.99\left(\mathrm{dd}, J_{1} 8.0 \mathrm{~Hz}, J_{2} 1.6 \mathrm{~Hz}, 1 \mathrm{H}, \mathrm{H}-5\right), 7.92\left(\mathrm{~s}, 1 \mathrm{H}, \mathrm{H}-3{ }^{\prime \prime}\right), 7.84\left(\mathrm{~d}, J 2.4 \mathrm{~Hz}, 1 \mathrm{H}, \mathrm{H}-1^{\prime}, \mathrm{NH}\right), 7.77(\mathrm{~m}, 1 \mathrm{H}$, H-7), 7.69 (dd, J $7.6 \mathrm{~Hz}, J_{2} 1.2 \mathrm{~Hz}, 1 \mathrm{H}, \mathrm{H}-5^{\prime}$ ), 7.48 (dt, J1 $\left.7.6 \mathrm{~Hz}, J_{2} 0.8 \mathrm{~Hz}, 1 \mathrm{H}, \mathrm{H}-6\right), 7.40$ (d, J $\left.8.4 \mathrm{~Hz}, 1 \mathrm{H}, \mathrm{H}-8\right)$, 7.34 (dt, J1 $\left.8.4 \mathrm{~Hz}, J_{2} 1.6 \mathrm{~Hz}, 1 \mathrm{H}, \mathrm{H}-7^{\prime}\right), 7.17$ (d, J $\left.8.4 \mathrm{~Hz}, 2 \mathrm{H}, \mathrm{H}-3^{\prime}, \mathrm{H}-5^{\prime}\right), 6.80(\mathrm{~m}, 4 \mathrm{H}), 6.40(\mathrm{~s}, 1 \mathrm{H}, \mathrm{H}-3), 5.93$ (d, J $\left.3.2 \mathrm{~Hz}, 1 \mathrm{H}, \mathrm{H}-2^{\prime}\right)$ and $5.26 \mathrm{ppm}\left(\mathrm{s}, 2 \mathrm{H}, \mathrm{H}-4{ }^{\prime} \mathrm{a}\right) .{ }^{13} \mathrm{C}$ NMR (100 MHz, DMSO- $\left.d_{6}\right): \delta_{\mathrm{c}} 176.8$ (C-4, C=O), 165.2 (C-2), 162.4 (C-4', C=O, amide), 155.6 (C-8a), 151.5 (C-3"'), 147.4 (C-8'a), 146.3 (C aromatic), 143.7 (C-5"'), 134.5 (C aromatic), 134.0 (C-7'), 129.1 (C-3', C-5'), 127.5 (C aromatic), 127.0 (C aromatic), 125.7 (C aromatic), 124.9 (C 
aromatic), 123.2 (C-5), 118.4 (C aromatic), 118.2 (C aromatic), 114.7 (C aromatic), 114.2 (C aromatic), 112.3 (C-

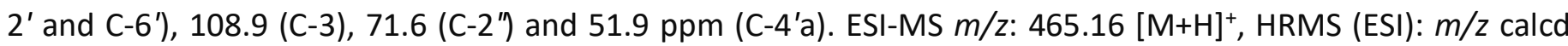
for $\mathrm{C}_{26} \mathrm{H}_{21} \mathrm{~N}_{6} \mathrm{O}_{3}[\mathrm{M}+\mathrm{H}]^{+}$: 465.1675; found: 465.1688 .

3-(\{4-[(1H-1,2,4-Triazol-1-yl)methyl]phenyl\}amino)-2-(3-bromo-4-oxo-4H-chromen-2-yl)-2,3-dihydroquinazolin-4(1H)-one (20b). Green crystalline solid (245 mg, 45\%). mp 260-264 ${ }^{\circ} \mathrm{C}$. IR (solid, $\mathrm{KBr}, v_{\max }, \mathrm{cm}^{-1}$ ): 3323 ( $\mathrm{NH}$, sec amine), 1736 ( $\mathrm{C}=\mathrm{O}$, ketone), 1675 (C=O, amide). ${ }^{1} \mathrm{H}$ NMR (400 MHz, DMSO- $\left.d_{6}\right): \delta_{\mathrm{H}} 8.59$ (s, 1H, H-3'a, $\mathrm{NH}$ ), 8.44 (s, 1H, H-5"'), 8.06 (dd, J1 $\left.8.0 \mathrm{~Hz}, J_{2} 1.2 \mathrm{~Hz}, 1 \mathrm{H}, \mathrm{H}-5\right), 7.93$ (s, 1H, H-3"'), 7.85 (d, J $\left.2.8 \mathrm{~Hz}, 1 \mathrm{H}, \mathrm{H}-1^{\prime}, \mathrm{NH}\right)$, $7.77\left(\mathrm{~m}, 2 \mathrm{H}, \mathrm{H}-7, \mathrm{H}-5^{\prime}\right), 7.52$ (t, J 7.6 Hz, 1H, H-6), 7.35 (dt, J1 $\left.8.8 \mathrm{~Hz}, J_{2} 2.0 \mathrm{~Hz}, 1 \mathrm{H}, \mathrm{H}-7^{\prime}\right), 7.15$ (d, J $8.0 \mathrm{~Hz}, 2 \mathrm{H}, \mathrm{H}-$ 3', H-5'), 7.06 (d, J $8.4 \mathrm{~Hz}, 1 \mathrm{H}, \mathrm{H}-8), 6.80\left(\mathrm{~m}, 4 \mathrm{H}, \mathrm{CH}\right.$ aromatic), 6.40 (d, $\left.2.4 \mathrm{~Hz}, 1 \mathrm{H}, \mathrm{H}-2^{\prime}\right)$ and $5.26 \mathrm{ppm}(\mathrm{s}, 2 \mathrm{H}, \mathrm{H}-$ 4'a). ${ }^{13} \mathrm{C}$ NMR (100 MHz, DMSO-d $d_{6}$ ): $\delta_{\mathrm{c}} 171.6$ (C-4, C=0), 163.0 (C-2), 161.4 (C-4', C=O amide), 154.3 (C aromatic), 151.5 (C-3"'), 147.1 (C aromatic), 146.0 (C aromatic), 143.8 (C-5"'), 135.2 (C aromatic), 134.0 (C-7'), 129.2 (C-3', C-5'), 127.3 (C aromatic), 127.1 (C-7), 126.3 (C-6), 125.6 (C aromatic), 121.2 (C-5), 118.1 (C aromatic), 118.0 (C aromatic), 114.3 (C aromatic), 113.8 (C aromatic), 112.2 (C-2' and C-6'), 108.0 (C-3), 72.0 (C-2') and 51.9 ppm (C-4'a). ESI-MS m/z: $543.07[\mathrm{M}+\mathrm{H}]^{+}, 545.07[\mathrm{M}+2+\mathrm{H}]^{+}, \mathrm{HRMS}(\mathrm{ESI}): \mathrm{m} / \mathrm{z}$ calcd for $\mathrm{C}_{26} \mathrm{H}_{20} \mathrm{BrN}_{6} \mathrm{O}_{3}[\mathrm{M}+\mathrm{H}]^{+}:$543.0780; found: 543.0799 .

3-(\{4-[(1H-1,2,4-Triazol-1-yl)methyl]phenyl\}amino)-2-(3-bromo-6-methyl-4-oxo-4H-chromen-2-yl)-2,3-dihydroquinazolin-4(1H)-one (20c). Pale brown crystalline solid $\left(278 \mathrm{mg}, 50 \%\right.$ ). mp 248-250 ${ }^{\circ} \mathrm{C}$. IR (solid, $\mathrm{KBr}, v_{\max }$, $\mathrm{cm}^{-1}$ ): 3252 ( $\mathrm{NH}$, sec amine), 1736 ( $\mathrm{C}=\mathrm{O}$, ketone), 1668 (C=O, amide). ${ }^{1} \mathrm{H}$ NMR (400 MHz, DMSO- $\left.d_{6}\right): \delta_{\mathrm{H}} 8.59$ (s, $\left.1 \mathrm{H}, \mathrm{H}-3{ }^{\prime} \mathrm{a}, \mathrm{NH}\right), 8.41\left(\mathrm{~s}, 1 \mathrm{H}, \mathrm{H}-5^{\prime \prime \prime}\right), 8.06(\mathrm{~s}, 1 \mathrm{H}, \mathrm{H}-5), 7.90\left(\mathrm{~s}, 1 \mathrm{H}, \mathrm{H}-3^{\prime \prime \prime}\right), 7.77$ (d, J 2.8 Hz, 1H, H-1', NH), 7.71 (d, J $7.6 \mathrm{~Hz}, 1 \mathrm{H}, \mathrm{H}-7$ ), 7.65 (d, $\left.8.4 \mathrm{~Hz}, 1 \mathrm{H}, \mathrm{H}-5^{\prime}\right), 7.31$ (dt, J1 $\left.8.8 \mathrm{~Hz}, J_{2} 2.0 \mathrm{~Hz}, 1 \mathrm{H}, \mathrm{H}-7^{\prime}\right), 7.15$ (d, J $8.0 \mathrm{~Hz}, 2 \mathrm{H}, \mathrm{H}-3^{\prime}, \mathrm{H}-$ $\left.5^{\prime}\right), 7.06(\mathrm{~d}, J 8.4 \mathrm{~Hz}, 1 \mathrm{H}, \mathrm{H}-8), 6.80\left(\mathrm{~m}, 4 \mathrm{H}, \mathrm{CH}\right.$ aromatic), 6.40 (d, $\left.2.4 \mathrm{~Hz}, 1 \mathrm{H}, \mathrm{H}-2^{\prime}\right), 5.25 \mathrm{ppm}\left(\mathrm{s}, 2 \mathrm{H}, \mathrm{H}-4{ }^{\prime} \mathrm{a}\right)$ and $2.49 \mathrm{ppm}(\mathrm{s}, 3 \mathrm{H}, \mathrm{H}-6 \mathrm{6}) .{ }^{13} \mathrm{C}$ NMR (100 MHz, DMSO- $\left.d_{6}\right): \delta_{c} 176.1$ (C-4, C=0), 164.9 (C-2), 162.3 (C=O amide), 155.2 (C-8a), 151.0 (C-3"), 147.1 (C-8'a), 145.9 (C aromatic), 143.3 (C-5"'), 134.5 (C aromatic), 134.1 (C-7'), 133.9 (C-6), 128.7 (C-3', C-5'), 127.1 (C aromatic), 126.9 (C aromatic), 124.9 (C aromatic), 123.2 (C-5), 117.9 (C aromatic), 118.2 (C aromatic), 114.7 (C aromatic), 114.3 (C aromatic), 112.3 (C-2' and C-6'), 108.9 (C-3), 71.4 (C-2'), 51.8 (C-4'a) and 29.9 ppm (C-6a). ESI-MS m/z: $557.09[\mathrm{M}+\mathrm{H}]^{+}, \mathrm{m} / \mathrm{z} 559.09[\mathrm{M}+2+\mathrm{H}]^{+}, \mathrm{HRMS}(\mathrm{ESI}): \mathrm{m} / \mathrm{z}$ calcd for $\mathrm{C}_{27} \mathrm{H}_{22} \mathrm{BrN}_{6} \mathrm{O}_{3}[\mathrm{M}+\mathrm{H}]^{+}:$557.0937; found: 557.0944 .

3-(\{4-[(1H-1,2,4-Triazol-1-yl)methyl]phenyl\}amino)-2-(6-chloro-4-oxo-4H-chromen-2-yl)-2,3-dihydroquinaz-

olin-4(1H)-one (20d). Yellow crystalline solid (224 mg, 45\%). mp 255-260 ${ }^{\circ} \mathrm{C}$. IR (solid, $\mathrm{KBr}, v_{\max }, \mathrm{cm}^{-1}$ ): 3269 ( $\mathrm{NH}$, sec amine), 1741 ( $\mathrm{C}=\mathrm{O}$, ketone), 1647 (C=O, amide). ${ }^{1} \mathrm{H}$ NMR (400 MHz, DMSO- $\left.d_{6}\right): \delta_{\mathrm{H}} 8.66$ (s, $1 \mathrm{H}, \mathrm{H}-3$ 'a, $\mathrm{NH}), 8.57$ (s, 1H, H-5"'), $7.92(\mathrm{~s}, 1 \mathrm{H}, \mathrm{H}-5), 7.91,\left(\mathrm{~s}, 1 \mathrm{H}, \mathrm{H}-3^{\prime \prime}\right), 7.84\left(\mathrm{~s}, 1 \mathrm{H}, \mathrm{H}-1^{\prime}, \mathrm{NH}\right), 7.82$ (d, J $\left.2.0 \mathrm{~Hz}, 1 \mathrm{H}, \mathrm{H}-7\right)$, 7.69 (d, J $\left.4.8 \mathrm{~Hz}, 1 \mathrm{H}, \mathrm{H}-5^{\prime}\right), 7.48$ (d, J $7.2 \mathrm{~Hz}, 1 \mathrm{H}, \mathrm{H}-8$ ), 7.34 (t, 1H, H-7'), 7.17 (d, J $\left.6.8 \mathrm{~Hz}, 2 \mathrm{H}, \mathrm{H}-3^{\prime}, \mathrm{H}^{\prime} 5^{\prime}\right), 6.80$ (m, 4H), $6.40(\mathrm{~s}, 1 \mathrm{H}, \mathrm{H}-3), 5.93\left(\mathrm{~d}, J 2.4 \mathrm{~Hz}, 1 \mathrm{H}, \mathrm{H}-2^{\prime}\right)$ and $5.26 \mathrm{ppm}\left(\mathrm{s}, 2 \mathrm{H}, \mathrm{H}-4{ }^{\prime} \mathrm{a}\right) .{ }^{13} \mathrm{C}$ NMR (100 MHz, DMSO$\left.d_{6}\right): \delta_{c} 175.8$ (C-4, C=O), 165.8 (C-2), 162.4 (C-4', C=O amide), 154.2 (C aromatic), 151.5 (C-3'), 147.4 (C aromatic), 146.3 (C aromatic), 143.7 (C-5'"), 134.5 (C aromatic), 134.1 (C-7'), 130.1 (C-6) 129.2 (C-3', C-5'), 127.6 (C aromatic), 127.1 (C-7), 124.3 (C aromatic), 123.9 (C-5), 120.9 (C aromatic), 118.3 (C aromatic), 114.8 (C aromatic), 114.2 (C aromatic), 112.3 (C-2' and C-6'), 108.9 (C-3), 71.5 (C-2') and 51.6 ppm (C-4'a). ESI-MS m/z: $499.12[\mathrm{M}+\mathrm{H}]+, \mathrm{m} / \mathrm{z} 501.12[\mathrm{M}+2+\mathrm{H}]^{+}$, HRMS (ESI): $\mathrm{m} / z$ calcd for $\mathrm{C}_{26} \mathrm{H}_{20} \mathrm{ClN}_{6} \mathrm{O}_{3}[\mathrm{M}+\mathrm{H}]^{+}$: 499.1285; found: 499.1290 . 


\section{Acknowledgements}

The authors are thankful to the management of Dr. Reddy's laboratories and Department of Chemistry, Osmania University for facilitating the research work.

\section{Supplementary Material}

Supplementary material $\left({ }^{1} \mathrm{H} N M R,{ }^{13} \mathrm{C}\right.$ NMR and HRMS spectrum for the compounds 11a, 15a, 19a and 20a) associated with this article can be found in the website.

\section{References}

1. Silva, C. F. M.; Pinto, D. C. G. A.; Silva, A. M. S. ChemMedChem 2016, 11, 2252. https://doi.org/10.1002/cmdc.201600359

2. Huang, W.; Liu, M. Z.; Li, Y.; Tan, Y.; Yang, G. F. Bioorg. Med. Chem. 2007, 15, 5191. https://doi.org/10.1016/j.bmc.2007.05.022

3. Gamal-Eldeen, A. M.; Djemgou, P. C.; Tchuendem, M.; Ngadjui, B. T.; Tane, P.; Toshifumi, H. Z Naturforsch. C 2007, 62, 331.

https://doi.org/10.1515/znc-2007-5-622

4. Duan, X.-F.; Zeng, J.; Lu, J.-W.; Zhang, Z.-B. J. Org. Chem. 2006, 71, 9873. https://doi.org/10.1021/jo061644d

5. Sadanadam, Y. S.; Reddy, R. M.; Bhaskar, R. A. Eur. J. Med. Chem. 1987, 22, 169. https://doi.org/10.1016/0223-5234(87)90015-8

6. Bonola, G.; Sianesi, E. J. Med. Chem. 1970, 13, 329.

https://doi.org/10.1021/jm00296a052

7. Gupta, R. C.; Nath, R.; Shanker, K.; Bhargava, K. P.; Kishore, K. J. Indian Chem. Soc. 1979, 56, 219.

8. $\quad$ Kung, P.-P.; Casper, M.D.; Cook, K.L.; Wilson-Lingardo, L.; Risen, L.M.; Vickers, T.A.; Ranken, R.; Blyn, L.B.; Wyatt, J.R. J. Med. Chem. 1999, 42, 4705.

https://doi.org/10.1021/jm9903500

9. Chinigo, M. G.; Paige, M.; Grindrod, S. J. Med. Chem. 2008, 51, 4620. https://doi.org/10.1021/jm800271c

10. Birch, H. L.; Buckley, G. M.; Davies, N.; Dyke, H. J.; Frost, E. J.; Gilbert, P. J.; Hannah, D. R.; Haughan, A. F.; Madigan, M. J.; Morgan, T.; Pitt, W. R.; Ratcliffe, A. J.; Ray, N. C.; Richard, M. D.; Sharpe, A.; Taylor, A. J.; Whitworth, J. M.; Williams, S. C. Bioorg. Med. Chem. Lett. 2005, 15, 5335. https://doi.org/10.1016/i.bmcl.2005.06.108

11. Hour, M. J.; Huang, L.-J.; Kuo, S.-C.; Xia, Y.; Bastow, K.; Nakanishi, Y.; Hamel, E.; Lee, K.-H. J. Med. Chem. 2000, 43, 4479.

https://doi.org/10.1021/im000151c 
12. Gangwal, N. A.; Kothawade, U. R.; Galande, A. D.; Pharande, D. S.; Dhake, A. S. Indian J. Heterocycl. Chem. 2001, 10, 291.

13. Rexall Drug Co., U.S. Patent 3257397, 1966.

14. Okumura, K.; Oine, T.; Yamada, Y.; Hayashi, G.; Nakama, M. J. Med. Chem. 1968, 11, 348. https://doi.org/10.1021/jm00308a036

15. Cohen, E.; Klarberg, B.; Vaughan, J. R. J. Am. Chem. Soc. 1959, 81, 5508. https://doi.org/10.1021/ja01529a062

16. Harlie, A.; Parish, J.; Gilliom, R. D. J. Med. Chem. 1982, 25, 98. https://doi.org/10.1021/im00343a022

17. Alagarsamy, V.; Solomon, V. R.; Murugan, M. Bioorg. Med. Chem. 2007, 15, 4009. https://doi.org/10.1016/i.bmc.2007.04.001

18. Levin, J. I.; Chan, P. I.; Bailey, T.; Katocs, A. S.; Venkatesan, A. M. Bioorg. Med. Chem. Lett. 1994, 4, 1141. https://doi.org/10.1016/S0960-894X(01)80244-4

19. Obaiah, N. N. K.; Raghavendra, M. G.; Pavankumar, S. C.; Rangappa, S. K.; Mantelingu, K. Eur. J. Chem. 2014, 5, 671.

https://doi.org/10.5155/eurjchem.5.4.671-675.1071

20. Kumar, A.; Tyagi, M.; Srivastava, V. K. Indian J. Chem. Sect B. 2003, 42, 2142.

21. Edgar, S. S.; Clifton, N. J. U. S. Patent 3265 697, 1966.

22. Mustazza, C.; Borioni, A.; Sestili, I.; Sbraccia, M.; Rodomonte, A.; Ferretti, R.; Giudice, M. R. D. Chem. Pharm. Bull. 2006, 54, 611.

https://doi.org/10.1248/cpb.54.611

23. Hirose, N.; Kuriyama, S.; Sohda, S.; Sakaguchi, K.; Yamamoto, H. Chem. Pharm. Bull. 1973, 21, 1005. https://doi.org/10.1248/cpb.21.1005

24. Bonola, G.; Da, R. P.; Magistretti, M. J.; Massarani, E.; Setnikar, I. J. Med. Chem. 1968, 11, 1136. https://doi.org/10.1021/im00312a007

25. Jolanta, O.; Kami,-S. K. Acta Polon. Pharm. Drug Res. 2006, 63, 101.

26. Mesbah, M. K. Egypt. J. Pharm. Sci. 1992, 33, 897.

27. Caldwell, E. Medical Express 2013.

28. Schwartz, H. J.; Blumenthal, M.; Brady, R.; Braun, S.; Lockey, R.; Myers, D.; Mansfield, L.; Mullarkey, M.; Owens, G.; Ratner, P.; Repsher, L.; Van, A. A. Chest 1996, 109, 945. https://doi.org/10.1378/chest.109.4.945

29. Chen, K.; Kuo, S.-C.; Hsieh, M. C.; Mauger, A.; Lin, C. M.; Hamel, E.; Lee, K.-H. J. Med. Chem. 1997, 40, 3049.

https://doi.org/10.1021/jm970146h

30. Ferrando, C.; Foy, J. M.; Pratt, C. N, Purvis, J. R. J. Pharm. Pharmacol. 1981, 33, 219. https://doi.org/10.1111/j.2042-7158.1981.tb13761.x

31. Ting, W.; Youxue, W.; Yasuhide, K.; Yoshinori, K.; Yuzo, S.; Nozomu, N.; Hitoshi, Y. Endocrinology 2008, 149, 358.

https://doi.org/10.1210/en.2007-0467

32. Solomon, V. R.; Hu, C.; Lee, H. Bioorg. Med. Chem. 2010, 18, 1563. https://doi.org/10.1016/j.bmc.2010.01.001

33. Kumar, R.; Kumar, A.; Jain, S.; Kaushik, D. Eur. J. Med. Chem. 2011, 46, 3543. https://doi.org/10.1016/i.ejmech.2011.04.035 
34. Domagala, J. M. J. Antimicrob. Chemother. 1994, 33, 685.

https://doi.org/10.1093/jac/33.4.685

35. Itoh, K.; Kuramoto, Y.; Amano, H.; Kazamori, D.; Yazaki, A. Eur. J. Med. Chem. 2015, $103,354$. https://doi.org/10.1016/j.ejmech.2015.08.015

36. Wang, Y.; Damu, G. L. V.; Lv, J. S.; Geng, R. X.; Yang, D. C.; Zhou, C. H. Bioorg. Med. Chem. Lett. 2012, 22, 5363.

https://doi.org/10.1016/i.bmcl.2012.07.064

37. Odagiri, T.; Inagaki, H.; Sugimoto, Y.; Nagamochi, M.; Miyauchi, R. N.; Kuroyanagi, J.; Kitamura, T.; Komoriya, S.; Takahashi, H. J. Med. Chem. 2013, 56, 974.

https://doi.org/10.1021/im301650g

38. Emami, S.; Shafiee, A.; Foroumadi, A. Mini Rev. Med. Chem. 2006, 6, 375. https://doi.org/10.2174/138955706776361493

39. Tsogoeva, S. B. Mini Rev. Med. Chem. 2010, 10, 773. https://doi.org/10.2174/138955710791608280

40. Uzma, S, Kalid, M. K.; Sridevi, C.; Taha, M.; Wadood, A.; Shantini, V.; Mehreen, G.; Perveen, S. Scientific Reports, 2017, 7, 16980.

https://doi.org/10.1038/s41598-017-17261-w

41. Terzidis, M. A.; Stephanidou-Stephanatou, J.; Tsoleridis, C. A. Tetrahedron Lett. 2009, 50, 1196. https://doi.org/10.1016/j.tetlet.2008.12.106

42. Alexandra, G.; Maria, J. M.; Jorge, G.; Eugenio, U.; Fernanda, B. J. Med. Chem. Rev. 2014, 114, 4960. https://doi.org/10.1021/cr400265z

43. Khilya, V. P.; Kupchevskaya, I. P.; Kazakov, A. L.; Tkachuk, T. M.; Golubushina, G. M. Chem. Heterocycl. Compd. 1982, 18, 240.

https://doi.org/10.1007/BF00522119

44. Karale, B. K.; Takate, S. J.; Salve, S. P.; Zaware, B. H.; Jadhav, S. S. Indian J. Chem. 2015, 54B, 798.

45. Venu, K.; Ramakrishna, G.; Anindita, C.; Muralidaran, K.; Satyanarayana, Y.; Manoranjan, B. J. Chem. Sciences. 2017, 129, 1233.

https://doi.org/10.1007/s12039-017-1328-9

46. Sabitha, G. Aldrichimica Acta. 1996, 29, 15.

47. Chandra, K. G.; Amarnath, C. Arkivoc 2015, vi, 288. http://dx.doi.org/10.3998/ark.5550190.p009.020

48. Jagadeesh, S. G.; Krupadanam, G. L. D.; Srimannrayana, G. Synth. Commun. 1998, $28,3827$. https://doi.org/10.1080/00397919808004936

49. Otto, D.; Gerda, V.; Otto, H. Eur. J. Org. chem. 1954, 16, 587.

50. Payard, M. and Couquelet, J. Synthesis, 1979, 889. https://doi.org/10.1055/s-1979-28862

51. Kamal, M. E.-S.; Ameen, M. A.; Abdel,-L. F. F.; Asmaa, H. M. Z. Naturforsch. 2012, 67b, 1144. 